\title{
On Realization Theory of Quantum Linear Systems *
}

\author{
John E. Gough ${ }^{\dagger} \quad$ Guofeng Zhang ${ }^{\ddagger}$
}

July 9,2018

\begin{abstract}
The purpose of this paper is to study the realization theory of quantum linear systems. It is shown that for a general quantum linear system its controllability and observability are equivalent and they can be checked by means of a simple matrix rank condition. Based on controllability and observability a specific realization is proposed for general quantum linear systems in which an uncontrollable and unobservable subspace is identified. When restricted to the passive case, it is found that a realization is minimal if and only if it is Hurwitz stable. Computational methods are proposed to find the cardinality of minimal realizations of a quantum linear passive system. It is found that the transfer function of a quantum linear passive system $G$ can be written as a fractional form in terms of a matrix function $\Sigma$; moreover, $G$ is lossless bounded real if and only if $\Sigma$ is lossless positive real. A type of realization for multi-inputmulti-output quantum linear passive systems is derived, which is closely related to its controllability and observability decomposition. Two realizations, namely the independent-oscillator realization and the chain-mode realization, are proposed for single-input-single-output quantum linear passive systems, and it is shown that under the assumption of minimal realization, the independent-oscillator realization is unique, and these two realizations are related to the lossless positive real matrix function $\Sigma$.

Keyword: Quantum linear systems, realization theory, controllability, observability.
\end{abstract}

\section{Introduction}

Linear systems and signals theory has been very useful in the analysis and engineering of dynamical systems. Many fundamental notions have been proposed to characterize dynamical systems from a control-theoretic point of view. For example, controllability describes the ability of steering internal system states by external input, observability refers to the possibility of reconstructing the state-space trajectory of a dynamical system based on its external input-output data. Based on controllability and observability, Kalman canonical decomposition reveals the internal structure of a linear system. This, in particular minimal realization as a very convenient and yet quite natural assumption, is the basis of widely used model reduction methods such as balanced truncation and optimal Hankel norm approximation. Moreover, fundamental dissipation theory has been well established and has been proven very effective in control systems design. All of these have been well documented, see, e.g., Kwakernaak \& Sivan, 1972; Willems, 1972; Anderson \& Vongpanitlerd, 1973; Kailath, 1980; van der Schaft, 1996; Zhou, Doyle \& Glover, 1996.

In recent years there has been a rapid growth in the study of quantum linear systems. Quantum linear systems and signals theory has been proven very effective in the study of many quantum systems including quantum optical systems, optomechanical systems, cavity quantum electro-magnetic dynamical systems, atomic ensembles and quantum memories, see, e.g., Gardiner \& Zoller, 2000; Wall \& Milburn, 2008; Wiseman \& Milburn, 2010; Stockton, van Handel \& Mabuchi, 2004; Zhang, Chen, Bhattacharya, \& Meystre, 2010; Massel, et al., 2011; Matyas, et al., 2011; Tian, 2012; Zhang, et al., 2013;

*Corresponding author Guofeng Zhang. Tel. +852-2766-6936. Fax +852-2764-4382. Email: Guofeng.Zhang@polyu.edu.hk.

${ }^{\dagger}$ Institute of Mathematics and Physics, Aberystwyth University, Ceredigion SY23 3BZ, Wales UK. (e-mail: jug@aber.ac.uk).

$\ddagger$ Department of Applied Mathematics, The Hong Kong Polytechnic University, Hong Kong, China. (e-mail: Guofeng.Zhang@polyu.edu.hk). 
Hush, Carvalho, Hedges \& James, 2013. Because of its analytical and computational advantages, the linear setting always serves as an essential starting point for development of a more general theory.

Controllability and observability of quantum linear passive systems have been discussed in [Maalouf \& Petersen, 2011a; these two properties are used to establish the complex-domain bounded real lemma (Maalouf \& Petersen, 2011a, Theorem 6.5]) for quantum linear passive systems, which is the basis of quantum $H^{\infty}$ coherent feedback control of quantum linear passive systems, Maalouf \& Petersen, 2011b. For a quantum linear passive system it is shown in Guta \& Yamamoto, 2013 Lemma 3.1] that controllability is equivalent to observability; moreover, a minimal realization is necessarily Hurwitz stable, Guta \& Yamamoto, 2013, Lemma 3.2]. In this paper we explore further controllability and observability of quantum linear systems. For general quantum linear systems (not necessarily passive), we show that controllability and observability are equivalent (Proposition 2.3). Moreover, a simple matrix rank condition is established for checking controllability and observability. Base on this result, a realization of general quantum linear systems is proposed, in which the uncontrollable and unobservable subspace is identified (Theorem 2.6). Theorem 2.6 can be viewed as the complex-domain counterpart of Theorem 3.1 in Yamamoto, 2013 in the real domain. However, it is can be easily seen from the proof of Lemma 2.5 that the structure of the unitary transformation involved is better revealed in the complex domain. Restricted to the passive case, we show that controllability, observability and Hurwitz stability are equivalent to each other (Lemma 3.3). Thus, the realization of a quantum linear passive system is minimal if and only if it is Hurwitz stable (Theorem 3.5). We also derive formulas for calculating the cardinality of minimal realizations of a given quantum linear passive system (Proposition 3.7 for the single-input-single-output case and Proposition 3.8 for the multi-input-multi-output case). Finally we show how a given quantum linear system can be written as a fractional form in term of a matrix function $\Sigma$ (Proposition 2.8), and for the passive case show that a quantum linear passive system $G$ is lossless bounded real if and only if the corresponding $\Sigma$ is lossless positive real (Theorem 3.11).

The synthesis problem of quantum linear systems has been investigated in Nurdin, James \& Doherty, 2009, where they showed that a quantum linear system can always be realized by a cascade of one-degree-of-freedom harmonic oscillators with possible direct Hamiltonian couplings among them if necessary. Then in Nurdin, 2010 a necessary and sufficient condition is derived for the realizability of quantum linear systems via pure cascading only. For the passive case, it is shown in Petersen, 2011 that, under certain conditions on the system matrices, a minimal quantum linear passive system can be realized by a cascade of one-degree-of-freedom harmonic oscillators. These restrictions were removed in Nurdin, 2010 which proves that all quantum linear passive systems can be realised by pure cascading of one-degreeof-freedom harmonic oscillators. Model reduction of quantum linear systems has been studied in, e.g., Petersen, 2013, and Nurdin, 2013]. In this paper we propose several realizations of quantum linear passive systems. For the multiinput-multi-output (MIMO) case we show that the proposed realization has a close relationship with controllability and observably of the quantum linear passive system (Theorem 4.1). In the single-input-single-output (SISO) case, we propose two realizations, namely the independent-oscillator realization and the chain-mode realization (Theorem 4.3 and Theorem 4.7), and finally we show that if the system is Hurwitz stable, these two realizations are related to the lossless positive real $\Sigma$ mentioned in the previous paragraph (Theorem 4.10).

The rest of the paper is organized as follows. Section 2 studies general quantum linear systems; specifically, Subsection 2.1 briefly reviews quantum linear systems, Subsection 2.2 investigates their controllability and observability, and Subsection 2.3 presents a fractional form for transfer functions of quantum linear systems. Section 3 studies quantum linear passive systems, specifically, Subsection 3.1 introduces quantum linear passive systems, Subsection 3.2 investigates their Hurwitz stability, controllability and observability, Subsection 3.3 studies minimal realizations of quantum linear passive systems, and Subsection 3.4 proposes a fractional form for transfer functions of quantum linear passive systems. Section 4 investigates realizations of quantum linear passive systems; specifically, Subsection 4.1 proposes a realization for MIMO quantum linear passive systems, Subsections 4.2.1 and 4.2.2 propose an independent-oscillator realization and a chain-mode realization for SISO quantum linear systems respectively, and Subsection 4.2 .3 discusses the uniqueness of the independent-oscillator realization. Section 5 concludes this paper.

Notations. $\quad m$ is the number of input channels, and $n$ is the number of degrees of freedom of a given quantum 
linear system, namely, the number of system oscillators. Given a column vector of complex numbers or operators $x=\left[\begin{array}{lll}x_{1} & \cdots & x_{k}\end{array}\right]^{T}$, define $x^{\#}=\left[\begin{array}{lll}x_{1}^{*} & \cdots & x_{k}^{*}\end{array}\right]^{T}$, where the asterisk $*$ indicates complex conjugation or Hilbert space adjoint. Denote $x^{\dagger}=\left(x^{\#}\right)^{T}$. Furthermore, define a column vector $\breve{x}$ to be $\breve{x}=\left[x^{T} \quad\left(x^{\#}\right)^{T}\right]^{T}$. Let $I_{k}$ be an identity matrix and $0_{k}$ a zero square matrix, both of dimension $k$. Define $J_{k}=\operatorname{diag}\left(I_{k},-I_{k}\right)$. Then for a matrix $X \in \mathbb{C}^{2 j \times 2 k}$, define $X^{b}=J_{k} X^{\dagger} J_{j}$. Given two constant matrices $U, V \in \mathbb{C}^{r \times k}$, define $\Delta(U, V)=\left[U V ; V^{\#} U^{\#}\right]$. Given two operators $A$ and $B$, their commutator is defined to be $[A, B]=A B-B A$. "œ" means if and only if. Finally, $\operatorname{Spec}(X)$ denotes the set of all distinct eigenvalues of the matrix $X, \sigma(X)$ denotes the diagonal matrix with diagonal entries being the non-zero singular values of the matrix $X, \operatorname{Ker}(X)$ denotes the null space of the matrix $X$, and Range $(X)$ denotes the space spanned by the columns of the matrix $X$.

\section{Quantum linear systems}

We first introduce quantum linear systems in Subsection 2.1. then discuss their controllability and observability in Subsection 2.2 and finally study their transfer functions in Subsection 2.3

\subsection{Quantum linear systems}

In this subsection quantum linear systems are briefly described in terms of the $(S, L, H)$ language, Gough \& James, 2009 . More discussions on quantum linear systems can be found in, e.g., Gardiner \& Zoller, 2000; Wall \& Milburn, 2008; Wiseman \& Milburn, 2010; Doherty \& Jacobs, 1999; Zhang \& James, 2012; Tezak, et al., 2012.

An open quantum linear system $G$ studied in this paper consists of $n$ interacting quantum harmonic oscillators driven by $m$ input boson fields. Each oscillator $j$ has an annihilation operator $a_{j}$ and a creation operator $a_{j}^{*} ; a_{j}$ and $a_{j}^{*}$ are operators on the system space $\mathfrak{h}$ which is an infinite-dimensional Hilbert space. The operators $a_{j}, a_{k}^{*}$ satisfy the canonical commutation relations: $\left[a_{j}, a_{k}^{*}\right]=\delta_{j k}$. Denote $\mathbf{a} \equiv\left[\begin{array}{lll}a_{1} & \cdots & a_{n}\end{array}\right]^{T}$. Then the initial (that is, before the interaction between the system and the input boson fields) Hamiltonian $H$ can be written as $H=(1 / 2) \breve{\mathbf{a}}^{\dagger} \Omega \breve{\mathbf{a}}$, where $\breve{\mathbf{a}}=\left[\mathbf{a}^{T}\left(\mathbf{a}^{\#}\right)^{T}\right]^{T}$ as introduced in the Notations part, and $\Omega=\Delta\left(\Omega_{-}, \Omega_{+}\right) \in \mathbb{C}^{2 n \times 2 n}$ is a Hermitian matrix with $\Omega_{-}, \Omega_{+} \in \mathbb{C}^{n \times n}$. L in the $(S, L, H)$ language describes the coupling of the system harmonic oscillators to the input boson fields. The coupling is linear and can be written as $L=\left[C_{-} C_{+}\right] \breve{a}$ with $C_{-}, C_{+} \in \mathbb{C}^{m \times n}$. Finally, in the linear setting $S$ in the $(S, L, H)$ language is taken to be a constant unitary matrix in $\mathbb{C}^{m \times m}$.

Each input boson field $j$ has an annihilation operator $b_{j}(t)$ and a creation operator $b_{j}^{*}(t)$, which are operators on an infinite-dimensional Hilbert space $\mathfrak{F}$. Let $\mathbf{b}(t) \equiv\left[b_{1}(t) \cdots b_{m}(t)\right]^{T}$. The operators $b_{j}(t)$ and their adjoint operators $b_{j}^{*}(t)$ satisfy the following commutation relations:

$$
\left[b_{j}(t), b_{k}^{*}(r)\right]=\delta_{j k} \delta(t-r), \quad \forall j, k=1, \ldots, m, \forall t, r \in \mathbb{R} .
$$

For each $j=1, \ldots, m$, the $j$-th input field can also be represented in the integral form $B_{j}(t) \equiv \int_{0}^{t} b_{j}(r) d r$, whose Ito increment is $d B_{j}(t) \equiv B_{j}(t+d t)-B_{j}(t)$. Denote $B(t) \equiv\left[B_{1}(t) \cdots B_{m}(t)\right]^{T}$. The gauge process can be defined by $\Lambda_{j k}(t)=\int_{0}^{t} b_{j}^{*}(r) b_{k}(r) d r,(j, k=1, \ldots, m)$. The field studied in this paper is assumed to be canonical, that is, the field operators $B_{j}(t), B_{k}^{*}(t), \Lambda_{r l}(t)$ satisfy the following Ito table:

\begin{tabular}{l|llll}
$\times$ & $d B_{k}$ & $d \Lambda_{k l}$ & $d B_{l}^{*}$ & $d t$ \\
\hline$d B_{i}$ & 0 & $\delta_{i k} d B_{l}$ & $\delta_{i l} d t$ & 0 \\
$d \Lambda_{i j}$ & 0 & $\delta_{j k} d \Lambda_{i l}$ & $\delta_{j l} d B_{i}^{*}$ & 0 \\
$d B_{j}^{*}$ & 0 & 0 & 0 & 0 \\
$d t$ & 0 & 0 & 0 & 0
\end{tabular}

Under mild assumptions, the temporal evolution of the open quantum system $G$ can be described in terms of the following quantum stochastic differential equation (QSDE):

$$
d U(t)=\left\{-\left(L^{\dagger} L / 2+i H\right) d t+d B^{\dagger}(t) L-L^{\dagger} S d B(t)+\operatorname{Tr}\left[(S-I) d \Lambda^{T}(t)\right]\right\} U(t), \quad t>0,
$$


with $U(0)=I$ being the identity operator. Let $X$ be an operator on the system space $\mathfrak{h}$. Then the temporal evolution of $X$, denoted $X(t) \equiv U(t)^{*}(X \otimes I) U(t)$, is governed by the following QSDE:

$$
\begin{aligned}
d X(t)= & \mathcal{L}_{L, H}(X(t)) d t+d B^{\dagger}(t) S^{\dagger}(t)[X(t), L(t)]+\left[L^{\dagger}(t), X(t)\right] S(t) d B(t) \\
& +\operatorname{Tr}\left[\left(S^{\dagger}(t) X(t) S(t)-X(t)\right) d \Lambda^{T}(t)\right],
\end{aligned}
$$

where the Lindblad operator $\mathcal{L}_{L, H}(X(t))$ is

$$
\mathcal{L}_{L, H}(X(t)) \equiv-i[X(t), H(t)]+\frac{1}{2} L^{\dagger}(t)[X(t), L(t)]+\frac{1}{2}\left[L^{\dagger}(t), X(t)\right] L(t) .
$$

Note that $X(t)$ is an operator on the joint system-field space $\mathfrak{h} \otimes \mathfrak{F}$.

Let $b_{\text {out }, j}(t)$ denote the $j$-th field after interacting with the system, and $B_{\text {out }, j}(t) \equiv \int_{0}^{t} b_{\text {out }, j}(r) d r$. We have $B_{\text {out }, j}(t)=$ $U^{*}(t)\left(I \otimes B_{j}(t)\right) U(t)$. Denote $B_{\text {out }}(t) \equiv\left[B_{\text {out }, 1}(t), \cdots B_{\text {out }, m}(t)\right]^{T}$. Then in compact form the output field equation is

$$
d B_{\text {out }}(t)=L(t) d t+S d B(t)
$$

Substituting $H=(1 / 2) \breve{a}^{\dagger} \Omega \breve{a}$ and $L=\left[C_{-} C_{+}\right] \breve{a}$ into (2.3) we have a quantum linear system:

$$
\begin{aligned}
d \breve{\mathbf{a}}(t) & =\mathcal{A} \breve{\mathbf{a}}(t) d t+\mathcal{B} d \breve{B}(t), \\
d \check{B}_{\text {out }}(t) & =\mathcal{C} \breve{\mathbf{a}}(t) d t+\mathcal{D} d \breve{B}(t),
\end{aligned}
$$

in which

$$
\mathcal{A}=-\frac{1}{2} C^{b} C-i J_{n} \Omega, \mathcal{B}=-C^{b} \Delta\left(S, 0_{m \times m}\right), \mathcal{C}=\Delta\left(C_{-}, C_{+}\right) \equiv C, \mathcal{D}=\Delta\left(S, 0_{m \times m}\right) .
$$

Clearly, the quantum linear system is parameterized by constant matrices $S, C, \Omega$. In the sequel, we use the notation $G \sim(S, C, \Omega)$ for the quantum linear system (2.6)-(2.7) with parameters given in (2.8).

For notation's sake, we introduce the following definition.

Definition 2.1 (2.6)- (2.7) with parameters given in (2.8) is said to be the realization of the quantum linear system $G \sim(S, C, \Omega)$.

The constant matrices $\mathcal{A}, \mathcal{B}, \mathcal{C}, \mathcal{D}$ in (2.8) satisfy the following fundamental relations:

$$
\mathcal{A}+\mathcal{A}^{b}+\mathcal{C}^{b} \mathcal{C}=0, \mathcal{B}=-\mathcal{C}^{b} \mathcal{D}, \mathcal{D}^{b} \mathcal{D}=I_{2 m}
$$

These equations are often called physically realizability conditions of quantum linear systems. More discussions on physical realizability of quantum linear systems can be found in, e.g., James, Nurdin \& Petersen, 2008; Zhang \& James, 2011; Zhang \& James, 2012.

\subsection{Controllability and observability}

In this subsection we study controllability and observability of quantum linear systems introduced in Subsection 2.1.

Let $X$ be an operator on the system space $\mathfrak{h}$. Denote by $\langle X(t)\rangle$ the expected value of $X(t)$ with respect to the initial joint system-field state (which is a unit vector in the Hilbert space $\mathfrak{h} \otimes \mathfrak{F}$ ). Then (2.6)-(2.7) gives rise to the following classical linear system

$$
\begin{aligned}
\frac{d\langle\breve{\mathbf{a}}(t)\rangle}{d t} & =\mathcal{A}\langle\breve{\mathbf{a}}(t)\rangle+\mathcal{B}\langle\breve{\mathbf{b}}(t)\rangle, \\
\frac{d\left\langle\breve{\mathbf{b}}_{\text {out }}(t)\right\rangle}{d t} & =\mathcal{C}\langle\breve{\mathbf{a}}(t)\rangle+\mathcal{D}\langle\breve{\mathbf{b}}(t\rangle) .
\end{aligned}
$$

Definition 2.2 The quantum linear system $G \sim(S, C, \Omega)$ is said to be Hurwitz stable (resp. controllable, observable) if the corresponding classical linear system (2.10)-(2.11) is Hurwitz stable (resp. controllable, observable). 
Due to the special structure of quantum linear systems, we have the following result concerning their controllability and observability.

Proposition 2.3 Given a quantum linear system $G \sim(S, C, \Omega)$, the following statements are equivalent:

(i) $G$ is controllable;

(ii) $G$ is observable;

(iii) $\operatorname{rank}\left(\mathbf{O}_{s}\right)=2 n$, where

$$
\mathbf{O}_{s} \equiv\left[\begin{array}{c}
C \\
C J_{n} \Omega \\
\vdots \\
C\left(J_{n} \Omega\right)^{2 n-1}
\end{array}\right] .
$$

Proof. (i) $\Rightarrow$ (ii). We show this by contradiction. Assume $G$ is not observable. By the classical control theory (see. e.g., Zhou, Doyle \& Glover, 1996, Theorems 3.3]) there exist a scalar $\lambda$ and a non-zero vector $v \in \mathbb{C}^{2 n}$ such that $\mathcal{A} v=\lambda v$ and $\mathcal{C} v=0$. So $J_{n} \Omega v=i \lambda v$ and $C v=0$. Let $u=J_{n} v$ and $\mu=-\lambda^{*}$. Then $u^{\dagger} \mathcal{B}=-v^{\dagger} C^{\dagger} J_{m}=0$, and

$$
u^{\dagger} \mathcal{A}=-\left(J_{n} v\right)^{\dagger}\left(C^{b} C / 2+i J_{n} \Omega\right)=-\left(J_{n} v\right)^{\dagger} i J_{n} \Omega=-i v^{\dagger} \Omega=-\lambda^{*} v^{\dagger} J_{n}=\mu u^{\dagger} .
$$

By a standard result in classical control theory, (see. e.g., Zhou, Doyle \& Glover, 1996, Theorems 3.1]), G is not controllable. We reach a contradiction.

(ii) $\Rightarrow$ (i). This can be established by reversing the proof for (i) $\Rightarrow$ (ii).

(ii) $\Rightarrow$ (iii). Let $v \in \mathbb{C}^{2 n}$ such that $\mathbf{O}_{s} v=0$. Then $\mathcal{C} v=C v=0$ and $C\left(J_{n} \Omega\right)^{k} v=0, k=1, \ldots, 2 n-1$. Moreover,

$$
\begin{aligned}
\mathcal{C} \mathcal{A} v= & -C\left(C^{b} C / 2+i J_{n} \Omega\right) v=-i C J_{n} \Omega v=0, \\
\mathcal{C} \mathcal{A}^{2} v= & -C\left(C^{b} C / 2+i J_{n} \Omega\right)^{2} v=C\left(J_{n} \Omega\right)^{2} v=0, \\
& \vdots \\
\mathcal{C A}^{2 n-1} v= & C\left(J_{n} \Omega\right)^{2 n-1} v=0 .
\end{aligned}
$$

But by (ii) $G$ is observable, therefore $v=0$. (iii) is established.

(iii) $\Rightarrow$ (ii). This can be established by reversing the proof for (ii) $\Rightarrow$ (iii).

Proposition 2.3 tells us that the controllability and observability of a quantum linear system $G \sim(S, C, \Omega)$ are equivalent; moreover they can be determined by checking the rank of the matrix $\mathbf{O}_{s}$.

On the basis of Proposition 2.3. we have the following result about the uncontrollable and unobservable subspace of a quantum linear system.

Proposition 2.4 Let $\mathbf{C} \equiv\left[\begin{array}{llll}\mathcal{B} & \mathcal{A B} & \cdots & \mathcal{A}^{2 n-1} \mathcal{B}\end{array}\right]$ and $\mathbf{O} \equiv\left[\begin{array}{llll}\mathcal{C}^{T} & (\mathcal{C} \mathcal{A})^{T} & \cdots & \left(\mathcal{C A}^{2 n-1}\right)^{T}\end{array}\right]^{T}$ be the controllability and observability matrices of a quantum linear system $G \sim(S, C, \Omega)$ respectively. Then (in the terminology of modern control theory, Kwakernaak \& Sivan, 1972]; Anderson \& Vongpanitlerd, 1973]; Kailath, 1980]; Zhou, Doyle E3 Glover, 1996]) the following statements hold:

(i) The unobservable subspace is

$$
\operatorname{Ker}(\mathbf{O})=\operatorname{Ker}\left(\mathbf{O}_{s}\right),
$$

where $\operatorname{Ker}(X)$ denotes the null space of the matrix $X$, as introduced in the Notations part.

(ii) The uncontrollable subspace is

$$
\operatorname{Ker}\left(\mathbf{C}^{\dagger}\right)=\operatorname{Ker}\left(\mathbf{O}_{\mathbf{s}} J_{n}\right) .
$$


(iii) The uncontrollable and unobservable subspace is $\operatorname{Ker}\left(\mathbf{O}_{s}\right) \cap \operatorname{Ker}\left(\mathbf{O}_{s} J_{n}\right)$.

Proposition 2.4 can be established in the similar way as Proposition 2.3.

Propositions 2.3 and 2.4 appear purely algebraic. Nevertheless, they have interesting and important physical consequences. We begin with the following lemma.

Lemma 2.5 The dimension of the space $\operatorname{Ker}\left(\mathbf{O}_{s}\right) \cap \operatorname{Ker}\left(\mathbf{O}_{s} J_{n}\right)$ is even. Let the dimension of $\operatorname{Ker}\left(\mathbf{O}_{s}\right) \cap \operatorname{Ker}\left(\mathbf{O}_{s} \mathbf{J}_{n}\right)$ be $2 l$ for some nonnegative integer $l$. There exists a matrix $V=\left[\begin{array}{ll}V_{1} & V_{2}\end{array}\right]$ with $V_{1} \in \mathbb{C}^{2 n \times 2 l}$ and $V_{2} \in \mathbb{C}^{2 n \times 2(n-l)}$ such that

$$
\begin{aligned}
\operatorname{Range}\left(V_{1}\right) & =\operatorname{Ker}\left(\mathbf{O}_{s}\right) \cap \operatorname{Ker}\left(\mathbf{O}_{s} J_{n}\right), \\
V V^{\dagger}=V^{\dagger} V & =I_{2 n}, \\
V^{\dagger} J_{n} V & =\left[\begin{array}{cc}
J_{l} & 0 \\
0 & J_{n-l}
\end{array}\right] .
\end{aligned}
$$

The proof is given in the Appendix.

We are ready to state the main result.

Theorem 2.6 Let $V$ be the matrix defined in Lemma 2.5. If Range( $\left.V_{1}\right)$ is an invariant space under the linear transformation of $\Omega$, then the transformed system

$$
\left[\begin{array}{c}
\breve{a}_{D F} \\
\breve{a}_{D}
\end{array}\right] \equiv V^{\dagger} \breve{a}
$$

has the following realization:

$$
\begin{aligned}
d \breve{a}_{D F}(t) & =-i J_{l} V_{1}^{\dagger} \Omega V_{1} \breve{a}_{D F}(t) d t \\
d \breve{a}_{D}(t) & =-\left(\left(C V_{2}\right)^{b}\left(C V_{2}\right) / 2+i J_{n-l} V_{2}^{\dagger} \Omega V_{2}\right) \breve{a}_{D}(t) d t-\left(C V_{2}\right)^{b} \mathcal{D} d \check{B}(t), \\
d \check{B}_{\text {out }}(t) & =\left(C V_{2}\right) \breve{a}_{D}(t) d t+\mathcal{D} d \check{B}(t) .
\end{aligned}
$$

Proof. Because Range $\left(V_{1}\right)=\operatorname{Ker}\left(\mathbf{O}_{s}\right) \cap \operatorname{Ker}\left(\mathbf{O}_{s} J_{n}\right)$, the coupling operator of the transformed mode [ $\left.\breve{a}_{D F}^{T} \quad \breve{a}_{D}^{T}\right]^{T}$ is $\mathcal{C} V=\left[\begin{array}{ll}0 & C V_{2}\end{array}\right]$. Moreover, because Range $\left(V_{1}\right)$ is an invariant space under the linear transformation of $\Omega$, there exists a matrix $Y$ such that $\Omega V_{1}=V_{1} Y$. We have $V_{1}^{\dagger} \Omega V_{2}=Y^{\dagger} V_{1}^{\dagger} V_{2}=0$ where (2.16) is used. This, together with (2.17), gives

$$
V^{\dagger} J_{n} \Omega V=V^{\dagger} J_{n} V V^{\dagger} \Omega V=\left[\begin{array}{cc}
J_{l} V_{1}^{\dagger} \Omega V_{1} & 0 \\
0 & J_{n-l} V_{2}^{\dagger} \Omega V_{2}
\end{array}\right]
$$

That is, the transformed system with mode $\left[\begin{array}{cc}\breve{a}_{D F}^{T} & \breve{a}_{D}^{T}\end{array}\right]^{T}$ has the realization (2.18)-(2.20).

Remark 1. By (2.18), the modes $\breve{a}_{D F}$ evolve unitarily as an isolated system. In literature such isolated modes embedded in an open quantum system is often called decoherence-free modes, see, e.g., Ticozzi \& Viola, 2008, Ticozzi \& Viola, 2009, Yamamoto, 2013. Theorem 2.6 can be viewed as the complex-domain counterpart of Theorem 3.1 in Yamamoto, 2013 in the real domain. However, with the help of the matrix $\mathbf{O}_{s}$, matters are simplified; moreover, it can be seen from the proof of Lemma 2.5 in the Appendix that the structure of the unitary transformation matrix $V$ is better revealed with the help of $\mathbf{O}_{s}$ and in the complex domain.

Finally, from the proof of Lemma 2.5] it can be seen that the dimension of the space $\operatorname{Ker}(C)$ is also even. Moreover we have the following corollary which shows that under some conditions the unobservable and uncontrollable subspace is exactly $\operatorname{Ker}(C)$.

Corollary 2.7 Let the dimension of the space $\operatorname{Ker}(C)$ be $2 r$. Let a matrix $T \in \mathbb{C}^{2 n \times 2 r}$ be such that Range $(T)=\operatorname{Ker}(C)$. If $J_{n} T=T J_{r}$ and Range $(T)$ is an invariant space under the linear transformation of $\Omega$, then $\operatorname{Ker}(C)=\operatorname{Ker}\left(\mathbf{O}_{\mathbf{s}}\right) \cap$ $\operatorname{Ker}\left(\mathbf{O}_{\mathbf{s}} J_{n}\right)$. 
Proof. Clearly, $\operatorname{Ker}\left(\mathbf{O}_{s}\right) \cap \operatorname{Ker}\left(\mathbf{O}_{s} J_{n}\right) \subset \operatorname{Ker}\left(\mathbf{O}_{s}\right) \subset \operatorname{Ker}(C)$. Thus it is sufficient to show that $\operatorname{Ker}(C) \subset$ $\operatorname{Ker}\left(\mathbf{O}_{s}\right) \cap \operatorname{Ker}\left(\mathbf{O}_{s} J_{n}\right)$. However Range $(T)=\operatorname{Ker}(C)$, we show that $\operatorname{Range}(T) \subset \operatorname{Ker}\left(\mathbf{O}_{s}\right) \cap \operatorname{Ker}\left(\mathbf{O}_{s} J_{n}\right)$. Because Range $(T)$ is invariant with respect to a linear transformation $\Omega$, there exist matrix $Y$ such that $\Omega T=T Y$. This, together with $J_{n} T=T J_{r}$, gives $C\left(J_{n} \Omega\right) T=C T J_{r} Y=0$. Similarly, for all $k \geq 1, \mathcal{C}\left(J_{n} \Omega\right)^{k} T=0$. That is, $\mathbf{O}_{s} T=0$. Moreover, $\mathbf{O}_{s} J_{n} T=\mathbf{O}_{s} T J_{r}=0$. Consequently $\operatorname{Ker}(C)=\operatorname{Range}(T) \subset \operatorname{Ker}\left(\mathbf{O}_{s}\right) \cap \operatorname{Ker}\left(\mathbf{O}_{s} J_{n}\right)$. This together with $\operatorname{Ker}\left(\mathbf{O}_{s}\right) \cap \operatorname{Ker}\left(\mathbf{O}_{s} J_{n}\right) \subset \operatorname{Ker}(C)$ yields $\operatorname{Ker}(C)=\operatorname{Ker}\left(\mathbf{O}_{s}\right) \cap \operatorname{Ker}\left(\mathbf{O}_{s} J_{n}\right)$.

Corollary 2.7 can be regarded as the complex-domain counterpart of Proposition 3.1 in Yamamoto, 2013 in the real domain.

\subsection{Transfer functions}

In the frequency domain, the transfer function of the system $G \sim(S, C, \Omega)$ is defined to be

$$
G(s) \equiv \mathcal{D}+\mathcal{C}(s I-\mathcal{A})^{-1} \mathcal{B} .
$$

This transfer function has the following fundamental property, see, e.g., Zhang \& James, 2013, Eq. (24)]:

$$
G(i \omega)^{b} G(i \omega)=G(i \omega) G(i \omega)^{b}=I_{2 m}, \quad \forall \omega \in \mathbb{R} .
$$

Interestingly, the transfer function $G(s)$ of the quantum linear system $G \sim(S, C, \Omega)$ can be written into a fractional form.

Proposition 2.8 The transfer function $G(s)$ for a Hurwitz stable quantum linear system $G \sim(S, C, \Omega)$ can be written in the following fractional form

$$
G(s)=(I-\Sigma(s))(I+\Sigma(s))^{-1} \Delta(S, 0)
$$

where

$$
\Sigma(s) \equiv \frac{1}{2} C\left(s I+i J_{n} \Omega\right)^{-1} C^{b}, \quad \forall \operatorname{Re}[s]>0 .
$$

Proof. Because the system $G(s)$ is Hurwitz stable, all the eigenvalues of the matrix $A$ have strictly negative real part, therefore the matrix $s I-A$ is invertible for all $\operatorname{Re}[s]>0$. Moreover, for all $\operatorname{Re}[s]>0$, by the Woodbury matrix inversion formula,

$$
\begin{aligned}
(s I-\mathcal{A})^{-1} & =\left(s I+i J_{n} \Omega+\frac{1}{2} C^{b} C\right)^{-1} \\
& =\left(s I+i J_{n} \Omega\right)^{-1}-\frac{1}{2}\left(s I+i J_{n} \Omega\right)^{-1} C^{b}\left(I+\frac{1}{2} C\left(s I+i J_{n} \Omega\right)^{-1} C^{b}\right)^{-1} C\left(s I+i J_{n} \Omega\right)^{-1} .
\end{aligned}
$$

As a result, for all $\operatorname{Re}[s]>0$,

$$
\begin{aligned}
& I-C(s I-\mathcal{A})^{-1} C^{b} \\
= & I-C\left\{\left(s I+i J_{n} \Omega\right)^{-1}-\frac{1}{2}\left(s I+i J_{n} \Omega\right)^{-1} C^{b}\left(I+\frac{1}{2} C\left(s I+i J_{n} \Omega\right)^{-1} C^{b}\right)^{-1} C\left(s I+i J_{n} \Omega\right)^{-1}\right\} C^{b} \\
= & I-2 \Sigma(s)+2 \Sigma(s)(I+\Sigma(s))^{-1} \Sigma(s) \\
= & (I-\Sigma(s))(I+\Sigma(s))^{-1}
\end{aligned}
$$

with $\Sigma(s)$ as defined in (2.24). Consequently,

$$
G(s)=\left(I-C(s I-\mathcal{A})^{-1} C^{b}\right) \Delta(S, 0)=(I-\Sigma(s))(I+\Sigma(s))^{-1} \Delta(S, 0) .
$$

\section{Quantum linear passive systems}

In this section quantum linear passive systems are studied. This type of systems is introduced in Subsection 3.1 Stability, controllability and observability are investigated in Subsection 3.2 while minimal realizations of quantum linear passive systems are studied in Subsection 3.3. The relation between $G$ and $\Sigma$ in the passive setting is discussed in Subsection 3.4 


\subsection{Quantum linear passive systems}

If the matrices $C_{+}=0$ and $\Omega_{+}=0$, the resulting system, parameterized by matrices $S, C_{-}, \Omega_{-}$, is often said to be a quantum linear passive system. In this case, it can be described entirely in terms of annihilation operators. Actually a quantum linear passive system has the following form:

$$
\begin{aligned}
d \mathbf{a}(t) & =A \mathbf{a}(t)-C_{-}^{\dagger} S d B(t), \\
d B_{\text {out }}(t) & =C_{-} \mathbf{a}(t)+S d B(t) .
\end{aligned}
$$

in which $A \equiv-\frac{1}{2} C_{-}^{\dagger} C_{-}-i \Omega_{-}$.

In analog to Definition 2.1 for realization of general linear systems we introduce the following realization concept for passive linear systems.

Definition 3.1 (3.1)-(3.2) is said to be the realization of the quantum linear passive system $G \sim\left(S, C_{-}, \Omega_{-}\right)$.

Clearly, the transfer function of $G \sim\left(S, C_{-}, \Omega_{-}\right)$is

$$
G(s)=S-C_{-}(s I-A)^{-1} C_{-}^{\dagger} S .
$$

Define

$$
\Sigma(s) \equiv \frac{1}{2} C_{-}\left(s I+i \Omega_{-}\right)^{-1} C_{-}^{\dagger} .
$$

Then, in analog to Proposition 2.8, we have

$$
G(s)=(I-\Sigma(s))(I+\Sigma(s))^{-1} S .
$$

In the passive case, Eq. (2.22) reduces to

$$
G(i \omega)^{\dagger} G(i \omega)=G(i \omega) G(i \omega)^{\dagger}=I_{m}, \quad \forall \omega \in \mathbb{R} .
$$

Because deferent realizations may correspond to the same transfer function (3.3), we introduce the following concept.

Definition 3.2 Two realizations are said to be unitarily equivalent if there exists a unitary transformation which transforms one to the other.

Clearly, two unitarily equivalent realizations correspond to the same transfer function.

\subsection{Stability, controllability, and observability}

In this subsection we study stability of quantum linear passive systems. In particular, we show that a quantum linear passive system $G \sim\left(S, C_{-}, \Omega_{-}\right)$is Hurwitz stable if and only if it is observable and controllable.

Lemma 3.3 The following statements for a quantum linear passive system $G \sim\left(S, C_{-}, \Omega_{-}\right)$are equivalent:

(i) $G$ is Hurwitz stable;

(ii) $G$ is observable;

(iii) $G$ is controllable.

Proof. (i) $\rightarrow$ (ii). Clearly, $X=I_{n}>0$ is the unique solution to the following Lyapunov equation

$$
A^{\dagger} X+X A+C_{-}^{\dagger} C_{-}=0 .
$$


According to Zhou, Doyle \& Glover, 1996 Lemma 3.18], $\left(C_{-}^{\dagger} C_{-}, A\right)$ is observable, so $\left(C_{-}, A\right)$ is observable. That is, $G$ is observable.

(ii) $\rightarrow$ (i). Because $X=I_{n}>0$ is a solution to Eq. (3.7), $C_{-}^{\dagger} C_{-} \geq 0$ and $\left(C_{-}^{\dagger} C_{-}, A\right)$ is observable, by Zhou, Doyle \& Glover, 199 Lemma 3.19], $A$ is Hurwitz stable.

The equivalence between (ii) and (iii) has been established in Proposition 2.3.

Remark 2. An alternative proof of the equivalence between (ii) and (iii) is given in Guta \& Yamamoto, 2013, Lemma 3.1]. An alternative proof of (ii) $\rightarrow$ (i) is given in Guta \& Yamamoto, 2013, Lemma 3.2].

\subsection{Minimal realization}

In this subsection we study minimal realization of a given quantum linear passive system $G \sim\left(S, C_{-}, \Omega_{-}\right)$. We first introduce the concept of minimal realization.

Definition 3.4 If a quantum linear passive system $G \sim\left(S, C_{-}, \Omega_{-}\right)$is both controllable and observable, we say its realization (3.1)-(3.2) is a minimal realization.

The following result is an immediate consequence of Lemma 3.3 .

Theorem 3.5 (3.1)-(3.2) is a minimal realization of the quantum linear passive system $G \sim\left(S, C_{-}, \Omega_{-}\right)$if and only if it is Hurwitz stable.

In what follows we study the following problem concerning minimal realization.

Problem 3.6 Given a quantum linear passive system $G \sim\left(S, C_{-}, \Omega_{-}\right)$which may not be Hurwitz stable, it may have a subsystem $\left(S, C_{\min }, \Omega_{\min }\right)$ which is Hurwitz stable. In this case, let $n_{\min }$ be the number of system oscillators in the minimal realization of $\left(S, C_{\min }, \Omega_{\min }\right)$. How to compute $n_{\min }$ ?

\subsubsection{The single-input-single-output (SISO) case}

Given a SISO quantum linear passive system $G(s)$, let the spectral decomposition of $\Omega_{-}$be

$$
\Omega_{-}=\sum_{\omega \in \operatorname{spec}\left(\Omega_{-}\right)} \omega P_{\omega}
$$

where $P_{\omega}$ denotes the projection onto the eigenspace of the eigenvalue $\omega$ of $\Omega_{-}$. Define

$$
\sigma\left(\Omega_{-}, C_{-}\right) \equiv\left\{\omega \in \operatorname{spec}\left(\Omega_{-}\right): C_{-} P_{\omega} C_{-}^{\dagger} \neq 0\right\} .
$$

The following result shows that the size of the set $\sigma\left(\Omega_{-}, C_{-}\right)$is nothing but $n_{\min }$.

Proposition 3.7 Given a SISO quantum linear passive system $G \sim\left(S, C_{-}, \Omega_{-}\right)$, the number $n_{\min }$ of oscillators of a minimal realization $\left(S, C_{\text {min }}, \Omega_{\text {min }}\right)$ is equal to the size of the set $\sigma\left(\Omega_{-}, C_{-}\right)$defined in (3.8).

The proof is given in the Appendix.

\subsubsection{The multi-input-multi-output (MIMO) case}

The following result is the MIMO version of Proposition 3.7 .

Proposition 3.8 For a MIMO quantum linear passive system $G \sim\left(S, C_{-}, \Omega_{-}\right)$, let the distinctive eigenvalues of $\Omega_{-}$be $\omega_{1}, \ldots, \omega_{r}$, each with algebraic multiplicity $\tau_{i}$ respectively, $i=1, \ldots, r$. Define $\Lambda_{i}=\omega_{i} I_{\tau_{i}}, i=1, \ldots, r$. Assume

$$
\Omega_{-}=\left[\begin{array}{ccc}
\Lambda_{1} & & 0 \\
& \ddots & \\
0 & & \Lambda_{r}
\end{array}\right] .
$$


Accordingly partition $C_{-}=\left[\begin{array}{llll}C_{1} & C_{2} & \cdots & C_{r}\end{array}\right]$ with $C_{i}$ having $\tau_{i}$ columns, $i=1, \ldots, r$. Then

$$
n_{\min }=\sum_{i=1}^{r} \text { column } \operatorname{rank}\left[C_{i}\right]
$$

In particular, if $\tau_{i}=1$ for all $i=1, \ldots, r$, that $i s$, all poles of $\Omega_{-}$are simple poles, then

$$
n_{\min }=\left\{\omega_{i} \in \operatorname{spec}\left(\Omega_{-}\right): \operatorname{Tr}\left[C_{-} P_{\omega_{i}} C_{-}^{\dagger}\right] \neq 0\right\}
$$

as given in Proposition 3.7 .

The construction in Proposition 3.8 is essentially the Gilbert's realization. Its proof follows the discussions in Kailath, 1980, Sec. 6.1] or [Zhou, Doyle \& Glover, 1996, Sec. 3.7]. The details are omitted.

\section{$3.4 G$ and $\Sigma$}

In this subsection we explore a further relation between a quantum linear passive system $G \sim\left(S, C_{-}, \Omega_{-}\right)$and $\Sigma$ defined in (3.4).

We first review the notions of lossless bounded real and lossless positive real. The bounded real lemma for quantum linear passive systems has been established in Maalouf \& Petersen, 2011a. Dissipation theory for more general quantum linear systems has been studied in James, Nurdin \& Petersen, 2008, Zhang \& James, 2011, while the nonlinear case has been studied in James \& Gough, 2010.

Definition 3.9 (Lossless Bounded Real, Maalouf \& Petersen, 2011a, Definition 6.3].) A quantum linear passive system $G=\left(S, C_{-}, \Omega_{-}\right)$is said to be lossless bounded real if it is Hurwitz stable and Eq. (3.6) holds.

According to Definition [3.9, a Hurwitz stable quantum linear passive system is naturally lossless bounded real, as derived in Maalouf \& Petersen, 2011a.

Positive real functions have been studied extensively in classical (namely, non-quantum) control theory, see, e.g., Anderson \& Vongpanitlerd, 1973. Here we state a complex-domain version of positive real functions.

Definition 3.10 (Lossless Positive Real.) A function $\Xi(s)$ is said to be positive real if it is analytic in $\operatorname{Re}[s]>0$ and satisfies

$$
\Xi(s)+\Xi(s)^{\dagger} \geq 0, \quad \forall \operatorname{Re}[s]>0 .
$$

Moreover, $\Xi(s)$ is called lossless positive real if is positive real and satisfies

$$
\Xi(i \omega)+\Xi(i \omega)^{\dagger}=0
$$

where $i \omega$ is not a pole of $\Xi(s)$.

The following result relates the lossless bounded realness of a quantum linear passive system $G \sim\left(S, C_{-}, \Omega_{-}\right)$to the lossless positive realness of $\Sigma(s)$ defined in Eq. (3.4).

Theorem 3.11 If a quantum linear passive system $G \sim\left(S, C_{-}, \Omega_{-}\right)$is minimal, then

(i) $G(s)$ is lossless bounded real.

(ii) $\Sigma(s)$ defined in Eq. 3.4 is lossless positive real. 
Proof. (i). Without loss of generality, assume $S=I_{m}$. Because $G \sim\left(I, C_{-}, \Omega_{-}\right)$is minimal, by Theorem 3.5 it is Hurwitz stable. Moreover, $G \sim\left(I, C_{-}, \Omega_{-}\right)$satisfies Eq. (3.6). Therefore, according to Definition 3.9, $G \sim\left(I, C_{-}, \Omega_{-}\right)$is lossless bonded real.

(ii). Assume $i \omega$ is not a pole of $\Sigma(s)$. Then the matrix $i \omega I+i \Omega_{-}$is invertible. Note that

$$
\begin{aligned}
\Sigma(s)+\Sigma(s)^{\dagger} & =\frac{1}{2} C_{-}\left(s I+i \Omega_{-}\right)^{-1} C_{-}^{\dagger}+\frac{1}{2} C_{-}\left(s^{*} I-i \Omega_{-}\right)^{-1} C_{-}^{\dagger} \\
& =\operatorname{Re}[s] C_{-}\left(s I+i \Omega_{-}\right)^{-1}\left(C_{-}\left(s I+i \Omega_{-}\right)^{-1}\right)^{\dagger}, \quad \forall \operatorname{Re}[s]>0 .
\end{aligned}
$$

By (3.12), $\Sigma(i \omega)+\Sigma(i \omega)^{\dagger}=0$. Therefore, by Definition 3.10 $\Sigma(s)$ is lossless positive real.

Remark 3. In fact it can be shown that in the minimal realization case (i) and (ii) in Theorem 3.11 are equivalent.

Remark 4. $\Sigma(s)$ is a linear port-Hamiltonian system, van der Schaft, 1996, Chapter 4]. However it is worth noting that a lossless positive real $\Sigma(s)$ may not generate a genuine quantum system $G(s)$ via $G(s)=(I-\Sigma(s))(I+\Sigma(s))^{-1}$. For example, given $\Sigma(s)=\frac{s^{2}+1}{s\left(s^{2}+2\right)}$, it is lossless positive real. However, it can be verified that $G(s)=(I-\Sigma(s))(I+\Sigma(s))^{-1}=$ $1-\frac{2\left(s^{2}+1\right)}{s^{3}+s^{2}+2 s+1}$ is not a genuine quantum linear system. Later in Section 4.2 .3 we will give an explicit form of $\Sigma(s)$ which generates a genuine quantum linear passive system $G$, see (4.28) for details.

Here we have used the annihilation-operator form to study dissipative properties of quantum linear passive systems. Because the resulting matrices may be complex-valued, they can be viewed as the complex versions of lossless bounded real and lossless positive real in terms of the quadrature form, James, Nurdin \& Petersen, 2008. In fact, if the quantum system is represented in the quadrature form, it is exactly the same lossless bounded real form as that in Anderson \& Vongpanitlerd, 1973, Secs. 2.6 and 2.7] for classical linear systems. In fact, the relation between lossless bounded real and lossless positive real is well-known in electric networks, see. e.g., Anderson \& Vongpanitlerd, 1973.

\section{Realizations for quantum linear passive systems}

Several realizations of quantum linear passive systems are proposed in this section. The multi-input-multi-output (MIMO) case is studied in Subsection 4.1. For the single-input-single-output (SISO) case, an independent-oscillator realization is proposed in Subsection 4.2.1. Fig. 2. a chain-mode realization is presented in Subsections 4.2.2. Fig. 3. and the uniqueness of the independent-oscillator realization is discussed in Subsection 4.2 .3

\subsection{Realizations for multi-input-multi-output models}

In this subsection a new realization for MIMO quantum linear passive systems is proposed.

Before presenting our realizations for quantum linear passive systems, we describe for completeness a realization proposed in Nurdin, 2010 and Petersen, 2011 using the series product to produce a realization of an $n$-oscillator system as a cascade of $n$ one-oscillator systems.

We begin with the observation that every matrix $n \times n$ matrix $A$ admits a Schur decomposition $A=U^{\dagger} A^{\prime} U$ with $U$ unitary and $A^{\prime}$ lower triangular. For a given quantum linear passive system $G \sim\left(S, C_{-}, \Omega_{-}\right)$, we define a unitary transform $a^{\prime} \equiv U a$, such that $A^{\prime}=U A U^{\dagger}$ is lower triangular. Accordingly denote $C^{\prime}=C_{-} U^{\dagger}$ and $\Omega^{\prime}=U \Omega_{-} U^{\dagger}$. The new system is thus $G^{\prime} \sim\left(S, C^{\prime}, \Omega^{\prime}\right)$. A standard result from linear systems theory shows that the two systems $G$ and $G^{\prime}$ have the same transfer function. In what follows we show the system $G^{\prime}$ has a cascade realization, Fig. 4.1. Because $A^{\prime}=-\frac{1}{2} C^{\prime \dagger} C^{\prime}-i \Omega^{\prime}$ is lower triangular, for $j<k$ we have $A_{j k}^{\prime}=-\frac{1}{2} C_{j}^{\prime \dagger} C_{k}^{\prime}-i \Omega_{j k}^{\prime}=0$, so $\Omega_{j k}^{\prime}=\frac{i}{2} C_{j}^{\prime \dagger} C_{k}^{\prime}$. Therefore the lower triangular components are

$$
A_{k j}^{\prime}=-\frac{1}{2} C_{k}^{\prime \dagger} C_{j}^{\prime}-i \Omega_{k j}^{\prime}=-\frac{1}{2} C_{k}^{\prime \dagger} C_{j}^{\prime}-i \Omega_{j k}^{\prime *} \equiv-C_{k}^{\prime \dagger} C_{j}^{\prime} .
$$

Let us now set $G_{0} \sim(S, 0,0)$ and $G_{k} \sim\left(I, C_{k}^{\prime}, \Omega_{k k}^{\prime}\right)$ then the new system $G^{\prime}$ has a the cascaded realization $G^{\prime}=G_{n} \triangleleft$ $\cdots \triangleleft G_{1} \triangleleft G_{0}$, Fig. 4.1, 


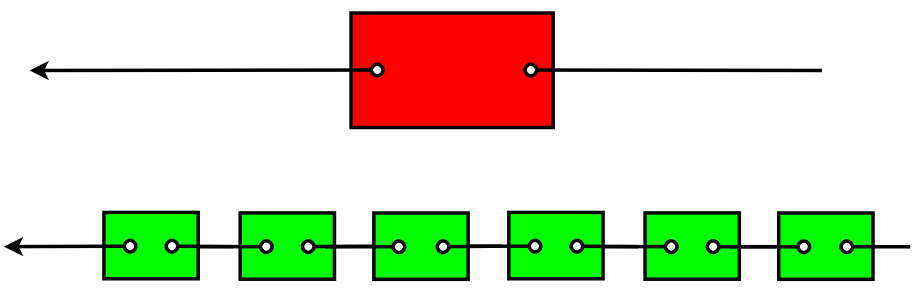

Figure 1: A quantum linear passive system with $n$ system oscillators is realised as a sequence of $n$ components in series, each one having a one-mode oscillator.

Next we present a new realization for MIMO quantum linear passive systems, which may have: 1) a set of interconnected principal oscillators $\tilde{a}_{\mathrm{pr}}$ that interact with the (possibly part of) environment $\left.\tilde{b}_{\mathrm{pr}}(t) ; 2\right)$ auxiliary oscillators $\tilde{a}_{\text {aux }, 1}$ and $\tilde{a}_{\text {aux }, 2}$ ) which only couple to the principal oscillators while otherwise being independent; 3 ) input-out channels $\tilde{b}_{\text {aux }}(t)$ that do not couple to the system oscillators.

Theorem 4.1 A quantum linear passive system $G=\left(I, C_{-}, \Omega_{-}\right)$can be unitarily transformed to another one with the corresponding realization

$$
\begin{aligned}
d \tilde{a}_{\mathrm{pr}}(t) & =-\left(\frac{\sigma\left(C_{-}\right)^{2}}{2}+i \tilde{\Omega}_{1}\right) \tilde{a}_{\mathrm{pr}}(t) d t-i \tilde{\Omega}_{21} \tilde{a}_{\mathrm{aux}, 1}(t) d t-i \tilde{\Omega}_{22} \tilde{a}_{\mathrm{aux}, 2}(t) d t-\sigma\left(C_{-}\right) d \tilde{B}_{\mathrm{in}, \mathrm{pr}}(t), \\
d \tilde{a}_{\mathrm{aux}, 1}(t) & =-i \sigma\left(\tilde{\Omega}_{3}\right) \tilde{a}_{\mathrm{aux}, 1}(t) d t-i \tilde{\Omega}_{21}^{\dagger} \tilde{a}_{\mathrm{pr}}(t) d t, \\
d \tilde{a}_{\mathrm{aux}, 2}(t) & =-i \tilde{\Omega}_{22}^{\dagger} \tilde{a}_{\mathrm{pr}}(t) d t, \\
d B_{\text {out }, \mathrm{pr}}(t) & =\sigma\left(C_{-}\right) \tilde{a}_{\mathrm{pr}}(t) d t+d B_{\mathrm{in}, \mathrm{pr}}(t), \\
d B_{\text {out }, \text { aux }}(t) & =d B_{\mathrm{in}, \text { aux }}(t),
\end{aligned}
$$

where $\tilde{\Omega}_{1}=\tilde{\Omega}_{1}^{\dagger}, \tilde{\Omega}_{3}=\tilde{\Omega}_{3}^{\dagger}$, and $\sigma(X)$ denotes the diagonal matrix with diagonal entries being the non-zero singular values of the matrix $X$. Clearly, this new realization corresponds the a quantum linear passive system $(I, \bar{C}, \bar{\Omega})$ with

$$
\bar{C} \equiv\left[\begin{array}{ccc}
\sigma\left(C_{-}\right) & 0 & 0 \\
0 & 0 & 0
\end{array}\right], \quad \bar{\Omega} \equiv\left[\begin{array}{ccc}
\tilde{\Omega}_{1} & \tilde{\Omega}_{21} & \tilde{\Omega}_{22} \\
\tilde{\Omega}_{21}^{\dagger} & \sigma\left(\tilde{\Omega}_{3}\right) & 0 \\
\tilde{\Omega}_{22}^{\dagger} & 0 & 0
\end{array}\right]
$$

The proof is given in the Appendix.

The realization (4.1)-(4.5) is in some sense like controllability and observability decomposition of quantum linear passive systems. In fact by Proposition 2.3 and Theorem 2.6. we have the following result.

Corollary 4.2 For the realization 4.1)- 4.5),

1. the mode $\tilde{a}_{\mathrm{pr}}$ is both controllable and observable;

2. if the system $G=\left(I, C_{-}, \Omega_{-}\right)$is Hurwitz stable, then $\tilde{\Omega}_{21} \neq 0$ and $\tilde{\Omega}_{22} \neq 0$.

Remark 5. When $m=1$, assuming minimal realization, from the proof given in the Appendix it can be seen that Theorem 4.1 reduces to Theorem 4.3 for the independent-oscillator realization of SISO systems to be discussed in Subsection 4.2.1.

\subsection{Realizations for single-input-single-output models}

In this subsection, two realizations, namely the independent-oscillator realization and the chain-mode realization, of SISO quantum linear passive systems are proposed. 


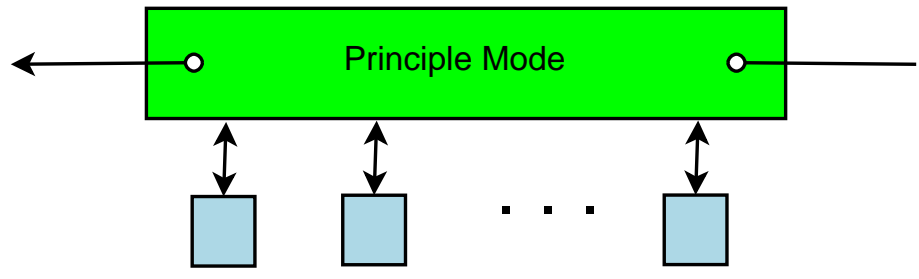

Figure 2: The independent-oscillator realization: the principal mode is coupled to $n-1$ independent auxiliary modes. The principal mode couples to the field, while the auxiliary modes are independent other than that they couple to the principal mode.

\subsubsection{Independent-oscillator realization}

Given a SISO quantum linear passive system $G \sim\left(I, C_{-}, \Omega_{-}\right)$where

$$
C_{-}=\left[\begin{array}{lll}
\sqrt{\gamma_{1}} & \cdots & \sqrt{\gamma_{n}}
\end{array}\right], \quad \Omega_{-}=\left(\omega_{j k}\right)_{n \times n}
$$

we show how to find a unitarily equivalent realization in terms of a single oscillator (the coupling mode $c_{0}$, we also call it the principle mode) which is then coupled to $n-1$ auxiliary modes $c_{1}, \cdots, c_{n-1}$. The auxiliary modes are themselves otherwise independent oscillators, Fig. 2.

Theorem 4.3 There exists a unitary matrix $T$ such that the transformed modes

$$
\mathbf{c}=\left[\begin{array}{c}
c_{0} \\
c_{1} \\
\vdots \\
c_{n-1}
\end{array}\right] \equiv T \mathbf{a}
$$

have the following realizations

$$
\begin{aligned}
d c_{0}(t) & =-\left(\gamma / 2+i \omega_{0}\right) c_{0}(t) d t-\sum_{j=1}^{n-1} i \sqrt{\kappa_{j}} c_{j}(t)-\sqrt{\gamma} d B(t), \\
d c_{j}(t) & =-i \omega_{j} c_{j}(t) d t-i \sqrt{\kappa_{j}} c_{0}(t) d t, \quad j=1, \ldots, n-1, \\
d B_{\text {out }}(t) & =\sqrt{\gamma} c_{0}(t)+d B(t),
\end{aligned}
$$

where

$$
\gamma \equiv \sum_{j=1}^{n} \gamma_{j}, \quad \omega_{0} \equiv \frac{1}{\gamma} \sum_{j, k=1}^{n} \sqrt{\gamma_{j} \gamma_{k}} \omega_{j k}
$$

and the other parameters $\omega_{j}, \kappa_{j}(j=1, \ldots, n-1)$ are given in the proof.

Proof. Let $R$ be a unitary matrix whose first row is $R_{1 j}=\sqrt{\gamma_{j} / \gamma},(j=1, \ldots n)$. Set $b_{j}^{\prime} \equiv \sum_{k=1}^{n} R_{j k} a_{k}, j=1, \ldots n$. We have $\left[b_{j}^{\prime}, b_{k}^{\prime *}\right]=\delta_{j k}$. Clearly $L=C_{-} \mathbf{a}=\sqrt{\gamma} b_{1}^{\prime}$ and $\left[L, b_{j}^{*}\right]=0$ for $j=2, \ldots$. Let us apply a further unitary transformation $V$ of the form $V=\left[\begin{array}{cc}1 & 0_{n-1}^{\top} \\ 0_{n-1} & \tilde{V}\end{array}\right]$ with $0_{n-1}$ the column vector of length $n-1$ with all zero entries and $\tilde{V}$ unitary in $\mathbb{C}^{(n-1) \times(n-1)}$ to be specified later. We set

$$
\mathbf{c}=\left[\begin{array}{c}
c_{0} \\
c_{1} \\
\vdots \\
c_{n-1}
\end{array}\right] \equiv V \mathbf{b}^{\prime}=V R \mathbf{a}
$$


We have $L=\sqrt{\gamma} c_{0}$. The Hamiltonian takes the form $H=\mathbf{c}^{\dagger} V R \Omega R^{\dagger} V^{\dagger} \mathbf{c}=\mathbf{c}^{\dagger} \Omega^{\prime} \mathbf{c}$, where

$$
\Omega^{\prime} \equiv\left[\begin{array}{cc}
1 & 0_{n-1}^{\top} \\
0_{n-1} & \tilde{V}
\end{array}\right] R \Omega_{-} R^{\dagger}\left[\begin{array}{cc}
1 & 0_{n-1}^{\top} \\
0_{n-1} & \tilde{V}^{\dagger}
\end{array}\right] .
$$

As the matrix $\tilde{V}$ is still arbitrary except being unitary, we may choose it to diagonalize the lower right $(n-1) \times(n-1)$ block of $R \Omega R^{\dagger}$, and with this choice we obtain $\Omega^{\prime}$ of the form

$$
\Omega_{\mathrm{IO}} \equiv\left[\begin{array}{cccc}
\omega_{0} & \varepsilon_{1}^{*} & \cdots & \varepsilon_{n-1}^{*} \\
\varepsilon_{1} & \omega_{1} & & 0 \\
\vdots & & \ddots & \\
\varepsilon_{n-1} & 0 & & \omega_{n-1}
\end{array}\right]
$$

It can be readily verified that $\omega_{0}=\frac{1}{\gamma} \sum_{j k=1}^{n} \sqrt{\gamma_{j} \gamma_{k}} \omega_{j k}$. Set $T=V R$ and the overall unitary transform is thus $\mathbf{c}=T \mathbf{a}$. Finally we may absorb the phases of the $\varepsilon_{k}$ into the modes, so without loss of generality we may assume that they are real and non-negative, say $\varepsilon_{k} \equiv \sqrt{\kappa_{k}}$.

By Proposition 2.3 and Theorem 2.6, we have the following corollary:

Corollary 4.4 For the realization 44.8)-4.11) constructed in Proposition 4.3, if $\kappa_{j}=0,(j=1, \ldots, n-1$, ) then the mode $c_{j}$ is neither controllable nor observable.

Because the two realizations, $G \sim\left(I, C_{-}, \Omega_{-}\right)$with $C_{-}, \Omega_{-}$defined in (4.7) and that in (4.8)-(4.11), are unitarily equivalent, they have the same transfer function. In what follows we derive their transfer function.

The following lemma turns out to be useful.

Lemma 4.5 We have the algebraic identity that

$$
\left(\left[\begin{array}{cccc}
a_{0} & b_{1} & \cdots & b_{n} \\
b_{1} & a_{1} & \ddots & 0 \\
\vdots & & \ddots & \\
b_{n} & 0 & & a_{n}
\end{array}\right]^{-1}\right)_{\text {row 1,column } 1}=\frac{1}{a_{0}-\sum_{k=1}^{n} \frac{b_{k}^{2}}{a_{k}}}
$$

where $(X)_{\text {row } 1, \text { column } 1}$ means the entry on the intersection of the first row and first column of a constant matrix $X$.

The proof is given in the Appendix.

We are now ready to present the transfer function.

Corollary 4.6 The SISO quantum linear passive system $G \sim\left(I, C_{-}, \Omega_{-}\right)$with $C_{-}, \Omega_{-}$defined in 4.7$)$ has a transfer function of the form

$$
G(s)=1-\frac{\gamma}{s+\frac{1}{2} \gamma+i \omega_{0}+\sum_{k=1}^{n-1} \frac{\kappa_{k}}{s+i \omega_{k}}} .
$$

The proof follows Theorem 4.3 and Lemma 4.5

Remark 6. Theorem 4.3 gives an independent-oscillator realization of a quantum linear passive system, Fig. 2 . Unfortunately, because the unitary matrices $V$ and $R$ used in the proof of Theorem 4.3 are by no means unique, it is unclear whether this realization is unique or not, that is, whether the parameters $\omega_{i}$ and $\kappa_{j}$ are uniquely determined by the system parameters $\gamma_{i}$ and $\omega_{j k}$ in (4.7) or not. In Theorem 4.10 to be given in Subsection 4.2.3, we show that the independent-oscillator realization is unique under the assumption of minimal realization. 


\subsubsection{Chain-mode realization}

In the subsection we present the chain-mode realization of SISO quantum linear passive systems.

Let $G \sim\left(I, C_{\min }, \Omega_{\min }\right)$ be a Hurwitz stable SISO quantum linear system with $n_{\min }$ the number of system oscillators. We assume that $\Omega_{\min }$ is diagonal and the entries of $C_{\min }$ are non-negative; specifically,

$$
\overline{\mathbf{a}}=\left[\begin{array}{c}
\bar{a}_{1} \\
\vdots \\
\bar{a}_{n_{\min }}
\end{array}\right], \Omega_{\min }=\operatorname{diag}\left(\bar{\omega}_{1}, \cdots, \bar{\omega}_{n_{\min }}\right), C_{\min }=\left[\sqrt{\bar{\gamma}_{1}}, \cdots, \sqrt{\bar{\gamma}_{n_{\min }}}\right] .
$$

Remark \%. Because the matrix $\Omega_{\min }$ is Hermitian, it can always be diagonalized. Similarly by absorbing phases into system oscillators if necessary, the entries of the matrix $C_{\min }$ can be taken to be non-negative. Thus, given a Hurwitz stable quantum linear passive system, one can always unitarily transform it to another one corresponding to (4.15). Moreover, by Proposition 3.7 minimality requires that $\bar{\omega}_{j} \neq \bar{\omega}_{k}$ if $j \neq k$, and $\bar{\gamma}_{j} \neq 0, j=1, \ldots, n_{\min }$.

In what follows we unitarily transform the system $G \sim\left(I, \Omega_{\min }, C_{\min }\right)$ to a chain-mode realization of an assembly of interacting oscillators, Fig. 3 .

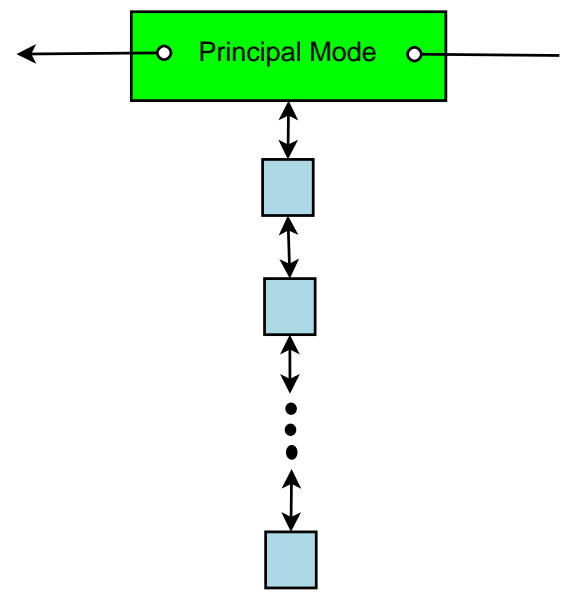

Figure 3: The Chain-mode realization: the principal mode is coupled to a non-damped mode which in turn is coupled to a finite chain of modes.

Theorem 4.7 For the system $G \sim\left(I, C_{\min }, \Omega_{\min }\right)$ defined by 4.15), there exists a unitary transform $W$ such that the transformed modes

$$
\left[\begin{array}{c}
\tilde{c}_{0} \\
\tilde{c}_{1} \\
\vdots \\
\tilde{c}_{n_{\min }-1}
\end{array}\right] \equiv W \overline{\mathbf{a}}
$$

have the following realization:

$$
\begin{aligned}
d \tilde{c}_{0}(t) & =-\left(\bar{\gamma} / 2+i \tilde{\omega}_{0}\right) \tilde{c}_{0}(t) d t-i \sqrt{\tilde{\kappa}_{1}} \tilde{c}_{1}(t) d t-\sqrt{\bar{\gamma}} d B(t), \\
d \tilde{c}_{j}(t) & =-i \tilde{\omega}_{j} \tilde{c}_{j}(t) d t-i \sqrt{\tilde{\kappa}_{j}} \tilde{c}_{j-1}(t) d t-i \sqrt{\tilde{\kappa}_{j+1}} \tilde{c}_{j+1}(t) d t, \quad j=1, \ldots, n_{\min }-2, \\
d \tilde{c}_{n_{\min }-1}(t) & =-i \tilde{\omega}_{n_{\min }-1} \tilde{c}_{n_{\min }-1}(t) d t-i \sqrt{\tilde{\kappa}_{n_{\min }-1}} \tilde{c}_{n_{\min }-2}(t) d t \\
d B_{\text {out }}(t) & =\sqrt{\bar{\gamma}} \tilde{c}_{0}(t) d t+d B(t),
\end{aligned}
$$

where the parameters $\tilde{\omega}_{j}$ and $\tilde{\kappa}_{j}$ are given respectively in (5.28) and (5.29) in the proof. 
The proof is given in the Appendix.

Remark 8. In the literature of continued fraction, Wall, 1948; Gautschi, 2004; Hughes, Christ \& Burghardt, 2009; Woods, et al., 2014, etc., the matrix

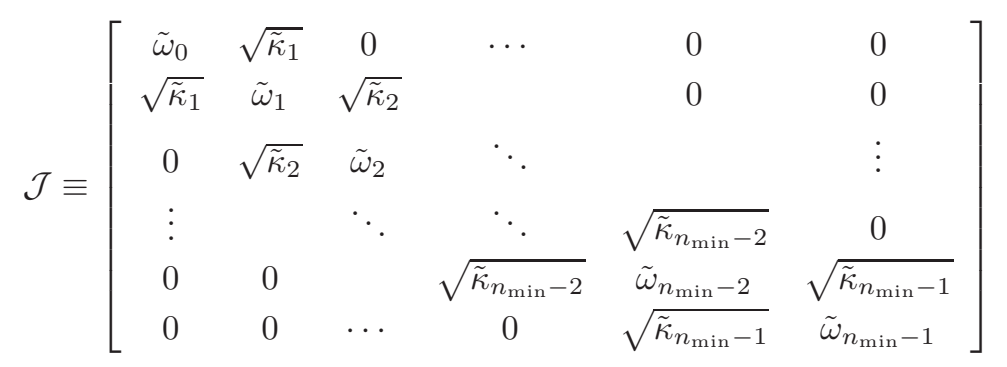

is often called a Jacob matrix. Clearly $\mathcal{J}$ is actually the Hamiltonian matrix for the new system corresponding to the realization (4.17)-(4.20).

Because the two realizations, $G \sim\left(I, C_{\min }, \Omega_{\min }\right)$ defined by (4.15) and that in (4.17)-(4.20), are unitarily equivalent, they share the same transfer function. Next we study their transfer function.

We begin with the following lemma.

Lemma 4.8 We have the algebraic identity that

$$
\left[\begin{array}{cccc}
a_{0} & b_{1} & & 0 \\
b_{1} & a_{1} & \ddots & \\
& \ddots & \ddots & b_{n} \\
0 & & b_{n} & a_{n}
\end{array}\right]_{\text {row 1,column 1 }}^{-1}=\frac{1}{a_{0}-\frac{b_{1}^{2}}{a_{1}-\frac{b_{2}^{2}}{a_{2}-} \cdot \frac{b_{n-1}^{2}}{a_{n-1}-\frac{b_{n}^{2}}{a_{n}}}}}
$$

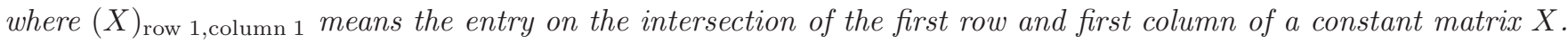

The proof is given in the Appendix.

Based on Theorem 4.7 and Lemma 4.8, we may derive the transfer function.

Corollary 4.9 The SISO quantum linear passive system $G \sim\left(I, C_{\min }, \Omega_{\min }\right)$ has a transfer function in the form of the continued fraction expansion

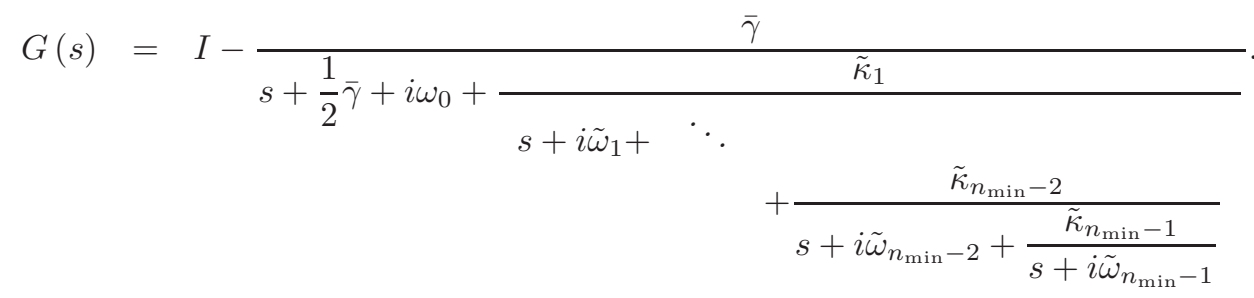

\subsubsection{Uniqueness of the independent-oscillator realization}

In Subsection 4.2.1 an independent-oscillator realization for SISO quantum linear passive systems is proposed. From the construction it is unclear whether the parameters in this independent-oscillator realization are unique, Remark 6 . In this subsection we show that they are indeed unique if minimality is assumed.

Theorem 4.10 Given a minimal quantum linear passive system $G \sim\left(I, C_{\min }, \Omega_{\min }\right)$ in [4.15), its unitarily equivalent independent-oscillator realization is unique. 
Proof. Firstly, for the minimal realization $G \sim\left(I, C_{\min }, \Omega_{\min }\right)$ in (4.15), by (4.12) and (5.30), $\omega_{0}=\tilde{\omega}_{0}$. Secondly, by (4.14) and (4.21) we see the transfer function takes the form

$$
G(s)=1-\frac{\gamma}{s+\frac{\gamma}{2}+i \omega_{0}+\Delta(s)}
$$

where

$$
\Delta(s) \equiv \sum_{k=1}^{n_{\min }-1} \frac{\kappa_{k}}{s+i \omega_{k}} \quad \begin{aligned}
& \tilde{\kappa}_{1} \\
& s+i \tilde{\omega}_{1}+\frac{\tilde{\kappa}_{2}}{s+i \tilde{\omega}_{2}+} \cdot \frac{\tilde{\kappa}_{n_{\min }-2}}{s+i \tilde{\omega}_{n_{\min }-2}+\frac{\tilde{\kappa}_{n_{\min }-1}}{s+\tilde{\omega}_{n_{\min }-1}}}
\end{aligned}
$$

in the independent-oscillator and chain-mode realizations respectively. Replacing $s$ with $i \omega$ in (4.22), (4.23) and (4.24) we have

$$
G(i \omega)=1+\frac{i \gamma}{\omega+\omega_{0}-\frac{\gamma}{2} i-\hat{\Delta}(\omega)}
$$

where

$$
\begin{aligned}
\hat{\Delta}(\omega) \equiv i \Delta(i \omega) & =\sum_{k=1}^{n_{\min }-1} \frac{\kappa_{k}}{\omega+\omega_{k}} \quad \frac{\tilde{\kappa}_{1}}{\omega+\tilde{\omega}_{1}-\frac{\tilde{\kappa}_{2}}{\omega+\tilde{\omega}_{2}-} \cdot \frac{\tilde{\kappa}_{n_{\min }-2}}{\omega+\tilde{\kappa}_{n_{\min }-1}}}
\end{aligned}
$$

in the independent-oscillator and chain-mode realizations respectively. By Theorem $4.7 \tilde{\omega}_{j}$ and $\tilde{\kappa}_{j}$ in (4.27) are uniquely determined by $C_{\min }$ and $\Omega_{\min }$, that is, $\hat{\Delta}(\omega)$ is unique. On the other hand, because $G=\left(I, C_{\min }, \Omega_{\min }\right)$ is minimal, in (4.26) $\omega_{j} \neq \omega_{k}$ if $j \neq k$, and $\kappa_{i} \neq 0$. Clearly, for this single pole fraction form of $\hat{\Delta}(\omega)$ in (4.26), $\kappa_{k}$ and $\omega_{k}$ are unique. The proof is completed.

We notice that (4.22) implies that

$$
\Sigma(s)=\frac{1}{2} \frac{\gamma}{s+i \omega_{0}+\Delta(s)}
$$

with $\Delta(s)$ given by (4.24).

Remark 9. Given $\Delta(s)$ in (4.23) and (4.24), by (4.28) an explicit form of $\Sigma(s)$ can be constructed, subsequently a quantum linear passive system $G(s)=(I-\Sigma(s))((I+\Sigma(s)))^{-1}$ can be constructed. According to (4.22), $G(s)$ constructed in this way is always a genuine quantum system. In this sense, (4.28) indicates what type of lossless positive real functions can generate a quantum linear passive system (which is lossless bounded real).

\section{Conclusion}

In this paper we have studied the realization theory of quantum linear systems. We have shown the equivalence between controllability and observability of general quantum linear systems, and in particular in the passive case they are equivalent to Hurwitz stability. Based on controllability and observability, a special form of realization has been proposed for 
general quantum linear systems which can be regarded as the complex-domain counterpart of the so-called decoherencefree subspace decomposition studied in Yamamoto, 2013. Specific to quantum linear passive systems, formulas for calculating the cardinality of minimal realizaitons are proposed. A specific realization is proposed for the multi-inputmulti-output case which is closely related to controllability and observability decomposition. Finally, two realizations, the independent-oscillator realization and the chain-mode realization, have been derived for the single-input-single-output case. It is expected that these results will find applications in quantum systems design.

\section{Acknowledgment}

The authors wish to thank Daniel Burgarth for pointing out Reference Woods, et al., 2014. The second author would like to thank Runze Cai, Lei Cui and Zhiyang Dong for helpful discussions.

\section{Appendix.}

Proof of Lemma 2.5. We first show that the dimension of the space $\operatorname{Ker}\left(\mathbf{O}_{s}\right) \cap \operatorname{Ker}\left(\mathbf{O}_{s} J_{n}\right)$ is even. If a nonzero vector

$$
v=\left[\begin{array}{l}
v_{1} \\
v_{2}
\end{array}\right] \in \operatorname{Ker}\left(\mathbf{O}_{s}\right) \cap \operatorname{Ker}\left(\mathbf{O}_{s} J_{n}\right)
$$

with $v_{1}, v_{2} \in \mathbb{C}^{n}$, then

$$
\mathcal{C}\left[\begin{array}{l}
v_{1} \\
v_{2}
\end{array}\right]=\left[\begin{array}{l}
C_{-} v_{1}+C_{+} v_{2} \\
C_{+}^{\#} v_{1}+C_{-}^{\#} v_{2}
\end{array}\right]=0, \quad \mathcal{C} J_{n}\left[\begin{array}{l}
v_{1} \\
v_{2}
\end{array}\right]=\left[\begin{array}{c}
C_{-} v_{1}-C_{+} v_{2} \\
C_{+}^{\#} v_{1}-C_{-}^{\#} v_{2}
\end{array}\right]=0,
$$

which are equivalent to

$$
\mathcal{C}\left[\begin{array}{cc}
v_{1} & 0 \\
0 & v_{2}
\end{array}\right]=0
$$

That is,

$$
\mathcal{C}\left[\begin{array}{l}
v_{1} \\
v_{2}
\end{array}\right]=0, \quad \mathcal{C} J_{n}\left[\begin{array}{l}
v_{1} \\
v_{2}
\end{array}\right]=0 \Longleftrightarrow \mathcal{C}\left[\begin{array}{c}
v_{1} \\
0
\end{array}\right]=0, \mathcal{C}\left[\begin{array}{c}
0 \\
v_{2}
\end{array}\right]=0 .
$$

On the other other hand, by (5.1) we also have

$$
\mathcal{C} J_{n} \Omega\left[\begin{array}{l}
v_{1} \\
v_{2}
\end{array}\right]=0, \quad \mathcal{C} J_{n} \Omega J_{n}\left[\begin{array}{l}
v_{1} \\
v_{2}
\end{array}\right]=0
$$

which are equivalent to

$$
\mathcal{C} J_{n} \Omega\left[\begin{array}{cc}
v_{1} & 0 \\
0 & v_{2}
\end{array}\right]=0
$$

Therefore we have

$$
\mathcal{C} J_{n} \Omega\left[\begin{array}{l}
v_{1} \\
v_{2}
\end{array}\right]=0, \quad \mathcal{C} J_{n} \Omega J_{n}\left[\begin{array}{l}
v_{1} \\
v_{2}
\end{array}\right]=0 \Longleftrightarrow \mathcal{C} J_{n} \Omega\left[\begin{array}{c}
v_{1} \\
0
\end{array}\right]=0, \quad \mathcal{C} J_{n} \Omega\left[\begin{array}{c}
0 \\
v_{2}
\end{array}\right]=0 .
$$

Analogously it can be shown that

$$
\mathcal{C}\left(J_{n} \Omega\right)^{k}\left[\begin{array}{l}
v_{1} \\
v_{2}
\end{array}\right]=0, \quad \mathcal{C} J_{n}\left(J_{n} \Omega\right)^{k}\left[\begin{array}{l}
v_{1} \\
v_{2}
\end{array}\right]=0 \Longleftrightarrow \mathcal{C}\left(J_{n} \Omega\right)^{k}\left[\begin{array}{c}
v_{1} \\
0
\end{array}\right]=0, \quad \mathcal{C}\left(J_{n} \Omega\right)^{k}\left[\begin{array}{c}
0 \\
v_{2}
\end{array}\right]=0, \quad k \geq 1
$$

(5.3), (5.5) and (5.6) indicate that

$$
v=\left[\begin{array}{l}
v_{1} \\
v_{2}
\end{array}\right] \in \operatorname{Ker}\left(\mathbf{O}_{s}\right) \cap \operatorname{Ker}\left(\mathbf{O}_{s} \mathbf{J}_{n}\right) \Longleftrightarrow\left[\begin{array}{c}
v_{1} \\
0
\end{array}\right],\left[\begin{array}{c}
0 \\
v_{2}
\end{array}\right] \in \operatorname{Ker}\left(\mathbf{O}_{s}\right) \cap \operatorname{Ker}\left(\mathbf{O}_{s} J_{n}\right) .
$$


Moreover, it can be readily shown that

$$
\begin{gathered}
{\left[\begin{array}{c}
v_{1} \\
0
\end{array}\right] \in \operatorname{Ker}\left(\mathbf{O}_{s}\right) \cap \operatorname{Ker}\left(\mathbf{O}_{s} J_{n}\right) \Longleftrightarrow\left[\begin{array}{c}
0 \\
v_{1}^{\#}
\end{array}\right] \in \operatorname{Ker}\left(\mathbf{O}_{s}\right) \cap \operatorname{Ker}\left(\mathbf{O}_{s} J_{n}\right),} \\
{\left[\begin{array}{c}
0 \\
v_{2}
\end{array}\right] \in \operatorname{Ker}\left(\mathbf{O}_{s}\right) \cap \operatorname{Ker}\left(\mathbf{O}_{s} J_{n}\right) \Longleftrightarrow\left[\begin{array}{c}
v_{2}^{\#} \\
0
\end{array}\right] \in \operatorname{Ker}\left(\mathbf{O}_{s}\right) \cap \operatorname{Ker}\left(\mathbf{O}_{s} J_{n}\right),}
\end{gathered}
$$

As a result, one can choose an orthonormal basis of $\operatorname{Ker}\left(\mathbf{O}_{s}\right) \cap \operatorname{Ker}\left(\mathbf{O}_{s} J_{n}\right)$ to be one of the form

$$
\left[\begin{array}{c}
v_{1} \\
0
\end{array}\right],\left[\begin{array}{c}
0 \\
v_{1}^{\#}
\end{array}\right], \cdots,\left[\begin{array}{c}
v_{l} \\
0
\end{array}\right],\left[\begin{array}{c}
0 \\
v_{l}^{\#}
\end{array}\right] .
$$

Therefore, the dimension of the space $\operatorname{Ker}\left(\mathbf{O}_{s}\right) \cap \operatorname{Ker}\left(\mathbf{O}_{s} J_{n}\right)$ is even. Here we take it to be $2 l$.

Secondly, we construct $V_{1} \in \mathbb{C}^{2 n \times 2 l}$. Noticing $\operatorname{Ker}\left(\mathbf{O}_{s} J_{n}\left[\begin{array}{cc}I_{n} & 0_{n}\end{array}\right]^{T}\right)=\operatorname{Ker}\left(\mathbf{O}_{s}\left[\begin{array}{ll}I_{n} & 0_{n}\end{array}\right]^{T}\right)$, we have

$$
\left[\begin{array}{c}
v_{i} \\
0
\end{array}\right] \in \operatorname{Ker}\left(\mathbf{O}_{s}\right) \cap \operatorname{Ker}\left(\mathbf{O}_{s} J_{n}\right) \Longleftrightarrow v_{i} \in \operatorname{Ker}\left(\mathbf{O}_{s}\left[\begin{array}{c}
I_{n} \\
0_{n}
\end{array}\right]\right) \text {. }
$$

Thus it is sufficient to construct the orthonormal basis vectors $v_{1}, \ldots, v_{l}$ for the space $\operatorname{Ker}\left(\mathbf{O}_{s}\left[\begin{array}{ll}I_{n} & 0_{n}\end{array}\right]^{T}\right)$. This can be done by the Gram-Schmidt orthogonalisation procedure. Define

$$
V_{1} \equiv\left[\begin{array}{cccccc}
v_{1} & \cdots & v_{l} & 0 & \cdots & 0 \\
0 & \cdots & 0 & v_{1}^{\#} & \cdots & v_{l}^{\#}
\end{array}\right] \in \mathbb{C}^{2 n \times 2 l} .
$$

For the above construction, Range $\left(V_{1}\right)=\operatorname{Ker}\left(\mathbf{O}_{s}\right) \cap \operatorname{Ker}\left(\mathbf{O}_{s} J_{n}\right)$. 2.15) is established.

Thirdly, we construct the matrix $V_{2}$. If a normalized vector $v_{l+1} \in \mathbb{C}^{n}$ such that for all $k=1, \ldots, l, v_{l+1}^{\dagger} v_{k}=0$, then $\left(v_{l+1}^{\#}\right)^{\dagger} v_{k}^{\#}=0$. That is, the normalized vectors $\left[\begin{array}{c}v_{l+1} \\ 0\end{array}\right]$ and $\left[\begin{array}{c}0 \\ v_{l+1}^{\#}\end{array}\right]$ are orthogonal to the space $\operatorname{Range}\left(V_{1}\right)$. Of course $\left[\begin{array}{c}v_{l+1} \\ 0\end{array}\right]$ and $\left[\begin{array}{c}0 \\ v_{l+1}^{\#}\end{array}\right]$ are orthogonal to each other too. By the Gram-Schmidt orthogonalisation procedure an orthonormal basis $\left\{v_{l+1}, \ldots, v_{n}\right\}$ can be found for the orthogonal space of the space spanned by the vectors $\left\{v_{1}, \ldots, v_{l}\right\}$. The an orthonormal matrix $V_{2}$ can be constructed to be

$$
V_{2} \equiv\left[\begin{array}{cccccc}
v_{l+1} & \cdots & v_{n} & 0 & \cdots & 0 \\
0 & \cdots & 0 & v_{l+1}^{\#} & \cdots & v_{n}^{\#}
\end{array}\right] \in \mathbb{C}^{2 n \times 2(n-l)} .
$$

Fourthly, define $V \equiv\left[\begin{array}{ll}V_{1} & V_{2}\end{array}\right]$. Clearly, $V^{\dagger} V=I_{2 n}$ which establishes (2.16).

Finally, because $V_{1}^{\dagger} J_{n}=J_{l} V_{1}^{\dagger}$, we have

$$
V^{\dagger} J_{n} V=\left[\begin{array}{cc}
V_{1}^{\dagger} J_{n} V_{1} & V_{1}^{\dagger} J_{n} V_{2} \\
V_{2}^{\dagger} J_{n} V_{1} & V_{2}^{\dagger} J_{n} V_{2}
\end{array}\right]=\left[\begin{array}{cc}
J_{l} & 0 \\
0 & J_{n-l}
\end{array}\right],
$$

which is (2.17). The proof is completed.

Proof of Proposition 3.7. Without loss of generality, assume that $\Omega_{-}$is diagonal. (Otherwise, there exists a unitary matrix $T$ such that $\bar{\Omega}=T \Omega_{-} T^{\dagger}$ is diagonal. Correspondingly, denote $\bar{P}_{\omega}=T P_{\omega} T^{\dagger}$ and $\bar{C}=C_{-} T^{\dagger}$. Then $\bar{C} \bar{P}_{\omega} \bar{C}^{\dagger}=C_{-} P_{\omega} C^{\dagger}$.) Let there be $r$ non-zero entries in the row vector $C_{-}$. Because $\Omega_{-}$is diagonal, if the $i$ th element of $C_{-}$is zero, then the $i$ th column of the matrix in (2.12) is a zero column. As a result, for minimality we need only consider non-zero elements of $C_{-}$. Without loss of generality, assume $C_{-}=\left[\begin{array}{lll}C_{1} & 0\end{array}\right]$, where $C_{1}=\left[\begin{array}{lllll}c_{1} & c_{2} & \cdots & c_{r}\end{array}\right]$ with $c_{i} \neq 0$, $(i=1, \ldots, r)$. Correspondingly, partition $\Omega_{-}$as

$$
\Omega_{-}=\left[\begin{array}{cc}
\Omega_{1} & 0 \\
0 & \Omega_{2}
\end{array}\right]
$$


where $\Omega_{1}$ is a $r \times r$ square diagonal matrix with $\omega_{1}, \ldots, \omega_{r}$ being diagonal entries. Clearly,

$$
\operatorname{rank}\left[\begin{array}{c}
C_{-} \\
C_{-} \Omega_{-} \\
\vdots \\
C_{-} \Omega_{-}^{n-1}
\end{array}\right]=\operatorname{rank}\left[\begin{array}{c}
C_{1} \\
C_{1} \Omega_{1} \\
\vdots \\
C_{1} \Omega_{1}^{r-1}
\end{array}\right]
$$

Notice that

$$
\left[\begin{array}{c}
C_{1} \\
C_{1} \Omega_{1} \\
\vdots \\
C_{1} \Omega_{1}^{r-1}
\end{array}\right]=\left[\begin{array}{cccc}
1 & 1 & \cdots & 1 \\
\omega_{1} & \omega_{2} & \cdots & \omega_{r} \\
\vdots & \vdots & \vdots & \vdots \\
\omega_{1}^{r-1} & \omega_{2}^{r-1} & \cdots & \omega_{r}^{r-1}
\end{array}\right]\left[\begin{array}{llll}
c_{1} & & & \\
& c_{2} & & \\
& & \ddots & \\
& & & c_{r}
\end{array}\right]
$$

According to Lemma 3.3 and noticing $c_{i} \neq 0$ for $i=1, \ldots, r$,

$$
n_{\min }=\operatorname{rank}\left[\begin{array}{c}
C_{1} \\
C_{1} \Omega_{1} \\
\vdots \\
C_{1} \Omega_{1}^{r-1}
\end{array}\right]=\operatorname{rank}\left[\begin{array}{cccc}
1 & 1 & \cdots & 1 \\
\omega_{1} & \omega_{2} & \cdots & \omega_{r} \\
\vdots & \vdots & \vdots & \vdots \\
\omega_{1}^{r-1} & \omega_{2}^{r-1} & \cdots & \omega_{r}^{r-1}
\end{array}\right] .
$$

Let $\ell$ be the total number of distinct diagonal entries of the matrix $\Omega_{1}$. By a property of the Vandermonde matrices, $\ell=n_{\text {min }}$. Finally, denote the distinct eigenvalues of $\Omega_{1}$ by $\hat{\omega}_{1}, \ldots, \hat{\omega}_{\ell}$. For each $i=1, \ldots, \ell$, because $c_{i} \neq 0, C_{-} P_{\hat{\omega}_{i}} C_{-}^{\dagger} \neq 0$. So we have shown that the number $n_{\min }$ of system oscillators of a minimal realization $\left(S, C_{\min }, \Omega_{\min }\right)$ equals the total number of elements of the set $\sigma\left(\Omega_{-}, C_{-}\right)$defined in (3.8).

Proof of Theorem 4.1. The proof can be done by construction. Let $\operatorname{rank}\left(C_{-}\right)=r>0$. Firstly, according to Bernstein, 2009, Theorem 5.6.4] there exist unitary matrices $R_{1} \in \mathbb{C}^{m \times m}$ and $R_{2} \in \mathbb{C}^{n \times n}$ such that

$$
R_{1} C_{-} R_{2}=\left[\begin{array}{cc}
\sigma\left(C_{-}\right)_{r \times r} & 0 \\
0 & 0
\end{array}\right]
$$

where $\sigma\left(C_{-}\right)$is a diagonal matrix with diagonal entries being singular values of the matrix $C_{-}$. Partition the matrix $R_{2}^{\dagger} \Omega_{-} R_{2}$ accordingly, and denote

$$
\bar{\Omega}=\left[\begin{array}{cc}
\tilde{\Omega}_{1} & \tilde{\Omega}_{2} \\
\tilde{\Omega}_{2}^{\dagger} & \tilde{\Omega}_{3}
\end{array}\right] \equiv R_{2}^{\dagger} \Omega_{-} R_{2}
$$

Define the unitary transformations

$$
\left[\begin{array}{c}
\tilde{b}_{\text {in }, \mathrm{pr}}(t) \\
\tilde{b}_{\text {in,aux }}(t)
\end{array}\right] \equiv R_{1} b(t), \quad\left[\begin{array}{c}
\tilde{b}_{\text {out }, \mathrm{pr}}(t) \\
\tilde{b}_{\text {out }, \text { aux }}(t)
\end{array}\right] \equiv R_{1} b_{\text {out }}(t), \quad\left[\begin{array}{c}
\tilde{a}_{\mathrm{pr}}(t) \\
a_{\text {aux }}(t)
\end{array}\right] \equiv R_{2}^{\dagger} a(t),
$$

where all the first blocks on the left-hand side are a row vector of dimension $r$. Then $G$ is unitarily equivalent to the following system

$$
\begin{aligned}
\dot{\tilde{a}}_{\mathrm{pr}} & =-\left(\sigma\left(C_{-}\right)^{2} / 2+i \tilde{\Omega}_{1}\right) \tilde{a}_{\mathrm{pr}}-i \tilde{\Omega}_{2} a_{\mathrm{aux}}-\sigma\left(C_{-}\right) \tilde{b}_{\mathrm{in}, \mathrm{pr}}(t), \\
\dot{a}_{\mathrm{aux}} & =-i \tilde{\Omega}_{2}^{\dagger} \tilde{a}_{\mathrm{pr}}-i \tilde{\Omega}_{3} a_{\mathrm{aux}}, \\
\tilde{b}_{\text {out }, \mathrm{pr}} & =\sigma\left(C_{-}\right) \tilde{a}_{\mathrm{pr}}+\tilde{b}_{\mathrm{in}, \mathrm{pr}}(t), \\
\tilde{b}_{\text {out }, \text { aux }} & =\tilde{b}_{\mathrm{in}, \text { aux }}(t) .
\end{aligned}
$$

By Schur decomposition there exists a unitary matrix $T \in \mathbb{C}^{(n-r) \times(n-r)}$ such that

$$
\tilde{\Omega}_{3}=T\left[\begin{array}{cc}
\sigma\left(\tilde{\Omega}_{3}\right) & 0 \\
0 & 0
\end{array}\right] T^{\dagger} .
$$


Accordingly, denote $\left[\begin{array}{ll}\tilde{\Omega}_{21} & \tilde{\Omega}_{22}\end{array}\right] \equiv \tilde{\Omega}_{2} T^{\dagger}$. As a result, applying the unitary transformation

$$
\left[\begin{array}{c}
\tilde{a}_{\mathrm{pr}} \\
\tilde{a}_{\mathrm{aux}, 1} \\
\tilde{a}_{\mathrm{aux}, 2}
\end{array}\right] \equiv\left[\begin{array}{cc}
I_{r \times r} & 0 \\
0 & T
\end{array}\right]\left[\begin{array}{c}
\tilde{a}_{\mathrm{pr}} \\
a_{\mathrm{aux}}
\end{array}\right]
$$

to (5.17)-(5.18) yields the final realization (4.1)-(4.5). Clearly the realization (4.1)-(4.5) corresponds to a quantum linear passive system whose the parameters are given in (4.6).

Proof of Lemma 4.5. We show this by induction. It is clear true for $n=1$, so we the assume it is true for a given $n$ and establish for $n+1$. Let us write $E_{11}(M)$ for the first entry (row 1, column 1 ) of a matrix $M$. Let us consider a sequence

$$
M_{n}=\left[\begin{array}{cccc}
a_{0} & b_{1} & \cdots & b_{n} \\
b_{1} & a_{1} & \ddots & 0 \\
\vdots & & \ddots & \\
b_{n} & 0 & & a_{n}
\end{array}\right]
$$

of matrices, then

$$
M_{n+1} \equiv\left[\begin{array}{cc}
M_{n} & b_{n+1} e_{n} \\
b_{n+1} e_{n}^{\top} & a_{n+1}
\end{array}\right], \text { where } e_{n}=\left[\begin{array}{c}
1 \\
0 \\
\vdots \\
0
\end{array}\right] \in \mathbb{C}^{n+1} .
$$

We recall the Schur-Feshbach inversion formula for a matrix in block form

$$
\left[\begin{array}{ll}
A_{11} & A_{12} \\
A_{21} & A_{22}
\end{array}\right]^{-1}=\left[\begin{array}{cc}
Y^{-1} & -Y^{-1} A_{12} A_{22}^{-1} \\
-A_{22}^{-1} A_{21} Y^{-1} & A_{22}^{-1}+A_{22}^{-1} A_{21} Y^{-1} A_{12} A_{22}^{-2}
\end{array}\right]
$$

where $Y=A_{11}-A_{21} A_{22}^{-1} A_{21}$. From the Schur-Feshbach formula we deduce that

$$
E_{11}\left(M_{n+1}^{-1}\right)=E_{11}\left(\left(M_{n}-\frac{b_{n+1}^{2}}{a_{n+1}} e_{n} e_{n}^{\top}\right)^{-1}\right) .
$$

However, the matrix $M_{n}-\left(b_{n+1}^{2} / a_{n+1}\right) e_{n} e_{n}^{\top}$ is identical to $M_{n}$ except that we replace the first row first column entry $a_{0}$ with $a_{0}-\left(b_{n+1}^{2} / a_{n+1}\right)$, and by assumption we should then have

$$
E_{11}\left(\left(M_{n}-\frac{b_{n+1}^{2}}{a_{n+1}} e_{n} e_{n}^{\top}\right)^{-1}\right)=\frac{1}{\left(a_{0}-\frac{b_{n+1}^{2}}{a_{n+1}}\right)-\sum_{k=1}^{n} \frac{b_{k}^{2}}{a_{k}}} .
$$

This establishes the formula for $n+1$, and so the formula is true by induction.

Proof of Proposition 4.7. The spectral distribution $\Phi$ associated with a SISO system $G \sim\left(S, C_{-}, \Omega_{-}\right)$is defined through the Stieltjes' integral, i.e.,

$$
\int_{-\infty}^{\infty} e^{i t \omega} d \Phi(\omega)=\frac{1}{C_{-} C_{-}^{\dagger}} C_{-} e^{i t \Omega_{-}} C_{-}^{\dagger},
$$

where the normalization coefficient $C_{-} C_{-}^{\dagger}>0$. In particular, in terms of the specific minimal realization $G \sim\left(S, C_{\min }, \Omega_{\min }\right)$ given in (4.15), we have

$$
d \Phi(\omega)=\sum_{j=1}^{n_{\min }} \frac{\bar{\gamma}_{j}}{\bar{\gamma}} \delta\left(\omega-\bar{\omega}_{j}\right) d \omega \equiv \bar{\mu}(\omega) d \omega
$$

where

$$
\bar{\gamma} \equiv \sum_{j=1}^{n_{\min }} \bar{\gamma}_{j}
$$


That is, the cardinality of the support of $d \Phi$ is exactly the number of oscillators $n_{\min }$ in the minimal realization of $G \sim\left(S, C_{\min }, \Omega_{\min }\right)$. The spectral distribution defined in (5.24) has only finitely many point supports. We define an inner product for polynomials in the field of real numbers in terms of this discrete spectral distribution. More specifically, given two real polynomials $P(\omega)$ and $Q(\omega)$, define their inner product with respect to $\bar{\mu}$ to be

$$
\langle P, Q\rangle_{\bar{\mu}} \equiv \int_{-\infty}^{\infty} P(\omega) Q(\omega) \bar{\mu}(\omega) d \omega=\sum_{j=1}^{n_{\min }} \frac{\bar{\gamma}_{j}}{\bar{\gamma}} P\left(\bar{\omega}_{j}\right) Q\left(\bar{\omega}_{j}\right) .
$$

The norm of a polynomial $P(\omega)$ is of course $\|P\| \equiv \sqrt{\langle P, P\rangle_{\bar{\mu}}}$. Next we introduce a sequence of $n_{\min }$ orthogonal polynomials $\left\{P_{i}\right\}$, which are defined via the Gram-Schmidt orthogonalization:

$$
P_{0}(\omega) \equiv 1, P_{j}(\omega)=\omega^{j}-\sum_{k=0}^{j-1} \frac{\left\langle\omega^{j}, P_{k}\right\rangle_{\bar{\mu}}}{\left\langle P_{k}, P_{k}\right\rangle_{\bar{\mu}}} P_{k}(\omega), \quad j=1, \ldots, n_{\min }-1,
$$

where $\left\langle\omega^{j}, P_{k}\right\rangle_{\bar{\mu}}$ is to be understood as $\left\langle\omega^{j}, P_{k}\right\rangle_{\bar{\mu}}=\int_{-\infty}^{\infty} \omega^{j} P_{k}(\omega) \bar{\mu}(\omega) d \omega$. It is easy to verify that the above orthogonal polynomial sequence $\left\{P_{j}\right\}_{j=0}^{n_{\min }}$ satisfies the following three-term recurrence relation, Gautschi, 2004, Theorem 1.27]

$$
P_{k+1}(\omega)=\left(\omega-\tilde{\omega}_{k}\right) P_{k}(\omega)-\sqrt{\tilde{\kappa}}_{k} P_{k-1}(\omega), \quad k=0, \ldots, n_{\min }-1,
$$

where $\tilde{\kappa}_{0} \equiv\left\|P_{0}\right\|$ and the convention $P_{-1} \equiv 0$ is assumed. Clearly,

$$
\tilde{\omega}_{k}=\frac{\left\langle\omega P_{k}, P_{k}\right\rangle_{\bar{\mu}}}{\left\langle P_{k}, P_{k}\right\rangle_{\bar{\mu}}}, \quad k=0, \ldots, n_{\min }-1,
$$

and

$$
\tilde{\kappa}_{k}=\sqrt{\frac{\left\langle P_{k}, P_{k}\right\rangle_{\bar{\mu}}}{\left\langle P_{k-1}, P_{k-1}\right\rangle_{\bar{\mu}}}}, \quad k=1, \ldots, n_{\min }-1 .
$$

(Note that $\tilde{\kappa}_{k} \neq 0, k=0, \ldots, n_{\min }-1$.) According to (5.28), we have

$$
\tilde{\omega}_{0}=\frac{1}{\bar{\gamma}} \sum_{j=1}^{n_{\min }} \bar{\gamma}_{j} \bar{\omega}_{j}
$$

By normalizing $\left\{P_{j}\right\}_{j=0}^{n_{\min }}$, that is define $\tilde{P}_{j} \equiv \frac{1}{\left\|P_{j}\right\|} P_{j}$, we can get a set of orthonormal polynomial sequence $\left\{\tilde{P}_{j}\right\}_{j=0}^{n_{\min }}$. We define a new set of oscillators to be

$$
\begin{aligned}
\tilde{c}_{0} & \equiv \sum_{j=1}^{n_{\min }} \sqrt{\frac{\bar{\gamma}_{j}}{\bar{\gamma}}} \tilde{P}_{0}\left(\bar{\omega}_{j}\right) \bar{a}_{j} \\
\tilde{c}_{k} & \equiv \sum_{j=1}^{n_{\min }} \sqrt{\frac{\bar{\gamma}_{j}}{\bar{\gamma}}} \tilde{P}_{k}\left(\bar{\omega}_{j}\right) \bar{a}_{j}, \quad k=1, \ldots, n_{\min }-1
\end{aligned}
$$

It can be verified that the transformation (5.31)-(5.32) is unitary. Moreover,

$$
\tilde{c}_{0}=\frac{1}{\sqrt{\bar{\gamma}}} \sum_{j=1}^{n_{\min }} \sqrt{\bar{\gamma}_{j}} \bar{a}_{j}
$$

and the canonical commutation relations $\left[\tilde{c}_{0}, \tilde{c}_{k}\right]=\left[\tilde{c}_{0}, \tilde{c}_{k}^{*}\right]=0,\left[\tilde{c}_{j}, \tilde{c}_{k}^{*}\right]=\delta_{j k}$ for $j, k=1, \ldots, n_{\min }-1$. By (5.33),

$$
\tilde{L}=\sqrt{\bar{\gamma}} \tilde{c}_{0} .
$$

Define matrices

$$
Q=\left[\begin{array}{ccc}
\tilde{P}_{0}\left(\bar{\omega}_{1}\right) & \cdots & \tilde{P}_{0}\left(\bar{\omega}_{n_{\min }}\right) \\
\vdots & \ddots & \vdots \\
\tilde{P}_{n_{\min }-1}\left(\bar{\omega}_{1}\right) & \cdots & \tilde{P}_{n_{\min }-1}\left(\bar{\omega}_{n_{\min }}\right)
\end{array}\right] \equiv\left[\begin{array}{c}
\tilde{P}_{0}(\bar{\omega}) \\
\vdots \\
\tilde{P}_{n_{\min }-1}(\bar{\omega})
\end{array}\right]
$$


and $\Gamma \equiv \operatorname{diag}\left(\sqrt{\frac{\bar{\gamma}_{1}}{\bar{\gamma}}}, \cdots, \sqrt{\frac{\bar{\gamma}_{\min }}{\bar{\gamma}}}\right)$. It can be shown that

$$
\sum_{k=0}^{n_{\min }-1} \frac{\bar{\gamma}_{i}}{\bar{\gamma}} \tilde{P}_{k}\left(\bar{\omega}_{i}\right) \tilde{P}_{k}\left(\bar{\omega}_{j}\right)=\delta_{i j}, \quad i, j=1, \ldots, n_{\min },
$$

see, e.g., Gautschi, 2004 Eq. (1.1.14)]. By (5.36), it can be verified that the inverse matrix of the matrix $Q$ turns out to be $Q^{-1}=\Gamma^{2}\left[\tilde{P}_{0}(\bar{\omega})^{\dagger} \ldots, \quad \tilde{P}_{n_{\min }-1}(\bar{\omega})^{\dagger}\right]$. Thus we have

$$
\left[\begin{array}{c}
\tilde{c}_{0} \\
\tilde{c}_{1} \\
\vdots \\
\tilde{c}_{n_{\min }-1}
\end{array}\right]=Q \Gamma\left[\begin{array}{c}
\bar{a}_{1} \\
\bar{a}_{2} \\
\vdots \\
\bar{a}_{n_{\min }}
\end{array}\right]
$$

With this, the Hamiltonian of the minimal realization can be re-written as

$$
\sum_{j=1}^{n_{\min }} \bar{\omega}_{j} \bar{a}_{j}^{*} \bar{a}_{j}=\left[\begin{array}{c}
\tilde{c}_{0} \\
\tilde{c}_{1} \\
\vdots \\
\tilde{c}_{n_{\min }-1}
\end{array}\right]^{\dagger}\left((Q \Gamma)^{-1}\right)^{\dagger} \Gamma\left[\begin{array}{ccc}
\bar{\omega}_{1} & & \\
& \ddots & \\
& & \bar{\omega}_{n_{\min }}
\end{array}\right](Q \Gamma)^{-1}\left[\begin{array}{c}
\tilde{c}_{0} \\
\tilde{c}_{1} \\
\vdots \\
\tilde{c}_{n_{\min }-1}
\end{array}\right] .
$$

Finally, according to (5.35) and (5.36), we have the new Hamiltonian matrix

$$
\begin{aligned}
& \tilde{H}=\left((Q \Gamma)^{-1}\right)^{\dagger} \Gamma\left[\begin{array}{ccc}
\bar{\omega}_{1} & & 0 \\
& \ddots & \\
0 & & \bar{\omega}_{n_{\min }}
\end{array}\right](Q \Gamma)^{-1}
\end{aligned}
$$

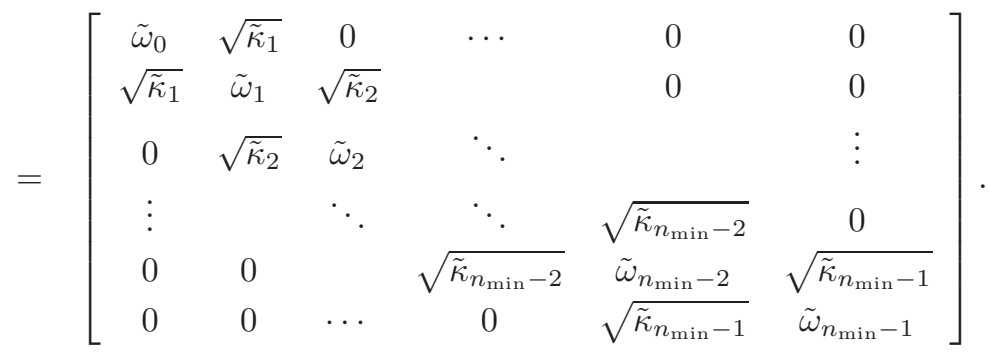

With the new coupling operator $\tilde{J}$ defined (5.34) and new Hamiltonian matrix $\tilde{H}$ defined in (5.39), the realization (4.17)-(4.20) can be obtained. The proof is completed.

Proof of Lemma 4.8. We again use induction. The formula is clearly true for $n=1$. Let us set

$$
N_{n}=\left[\begin{array}{cccc}
a_{0} & b_{1} & & 0 \\
b_{1} & a_{1} & \ddots & \\
& \ddots & \ddots & b_{n} \\
0 & & b_{n} & a_{n}
\end{array}\right]
$$

and so

$$
N_{n+1}=\left[\begin{array}{cc}
N_{n} & b_{n+1} f_{n} \\
b_{n+1} f_{n}^{\top} & a_{n+1}
\end{array}\right], \text { where } f_{n}=\left[\begin{array}{c}
0 \\
\vdots \\
0 \\
1
\end{array}\right] \in \mathbb{C}^{n+1}
$$


Let us write $E_{11}(M)$ for the first entry (row 1, column 1) of a matrix $M$. We deduce from the Schur-Feshbach formula (5.23) that

$$
E_{11}\left(N_{n+1}^{-1}\right)=E_{11}\left(\left(N_{n}-\frac{b_{n+1}^{2}}{a_{n+1}} f_{n} f_{n}^{\top}\right)^{-1}\right) .
$$

However, the matrix $N_{n}-\left(b_{n+1}^{2} / a_{n+1}\right) f_{n} f_{n}^{\top}$ is identical to $N_{n}$ except that we replace the last row, last column entry $a_{n}$ with $a_{n}-\left(b_{n+1}^{2} / a_{n+1}\right)$, and if by assumption the relation is true for $n$ we deduce the formula for $n+1$. The formula is true by induction.

\section{References}

[Anderson \& Vongpanitlerd, 1973] Anderson, B. D. O. \& Vongpanitlerd, S. (1973). Network Analysis and Synthesis. Prentice-Hall, Englewood Cliffs, NJ, (also, Dover edition, 2006).

[Bernstein, 2009] Bernstein, D. S. (2009). Matrix Mathematics, Theory,Facts, and Formulas. Princeton University Press, Princeton and Oxford.

[Doherty \& Jacobs, 1999] Doherty, A. \& Jacobs, K. (1999). Feedback-control of quantum systems using continuous stateestimation. Phys. Rev. A 60, 2700-2711.

[Gardiner \& Zoller, 2000] Gardiner, C. W. \& Zoller, P. (2000). Quantum Noise. Springer, Berlin.

[Gautschi, 2004] Gautschi, W. (2000). Orthogonal Polynomials Computation and Approximation. Oxford Science Publications.

[Gough \& James, 2009] Gough, J. E. \& James, M. R. (2009). The series product and its application to quantum feedforward and feedback networks. IEEE Trans. Automat. Contr. 54, 2530-2544.

[Guta \& Yamamoto, 2013] Guta M. \& Yamamoto N. (2013). Systems identification for passive linear quantum systems: the transfer function approach. arxiv: $1303.3771 v 1$.

[Hughes, Christ \& Burghardt, 2009] Hughes, K.H., Christ, C.D., \& Burghardt, I. (2009). Effective-mode representation of non-Markovian dynamics. J. Chem. Phys., 131, 024109, 2009.

[Hush, Carvalho, Hedges \& James, 2013] Hush, M., Carvalho, A. R. R, Hedges, M., \& James, M. R. (2013). Analysis of the operation of gradient echo memories using a quantum input-output model. New J. Phys. 15, 085020.

[James, Nurdin \& Petersen, 2008] James, M. R, Nurdin, H. I, \& Petersen, I. R. (2008). $H^{\infty}$ control of linear quantum stochastic systems. IEEE Trans. Automat. Contr. 53, 1787-1803.

[James \& Gough, 2010] James, M.R. \& Gough, J.E. (2010). Quantum dissipative systems and feedback control design by interconnection. IEEE Trans. Automat. Contrl. 55, 1806-1821.

[Kailath, 1980] Kailath, T. (1980). Linear Systems. Prentice-Hall, Inc, Englewood, Cliffs, N.J..

[Kwakernaak \& Sivan, 1972] Kwakernaak, H. \& Sivan, R. (1972). Linear OptimalControl Systems. John Wiley \& Sons, Inc.

[Maalouf \& Petersen, 2011a] Maalouf, A. \& Petersen, I. R. (2011). Bounded real properties for a class of linear complex quantum systems. IEEE Trans. Automat. Contr. 56, 786-801.

[Maalouf \& Petersen, 2011b] Maalouf, A. \& Petersen, I. R. (2011). Coherent $H^{\infty}$ control for a class of linear complex quantum systems. IEEE Trans. Automat. Contr. 56, 309-319. 
[Massel, et al., 2011] Massel, F., Heikkila, T.T., Pirkkalainen, J.-M., Cho, S.U., Saloniemi, H., Hakonen, P.J., \& Sillanpaa, M.A. (2011). Microwave amplification with nanomechanical resonators. Nature 480, 351-354.

[Matyas, et al., 2011] Matyas, A., Jirauschek, C., Peretti, F., Lugli, P., \& Csaba, G. (2011). Linear circuit models for on-chip quantum electrodynamics. IEEE Trans. Microwave Theory and Techniques 59, 65-71.

[Nurdin, James \& Doherty, 2009] Nurdin, H. I, James, M. R., \& Doherty, A. (2009). Network synthesis of linear dynamical quantum stochastic systems. SIAM J. Contr. and Optim. 48, 2686-2718.

[Nurdin, 2010] Nurdin, H.I. (2010). On synthesis of linear quantum stochastic systems by pure cascading. IEEE Trans. Automat. Contr. 55,2439-2444.

[Nurdin, 2013] Nurdin, H.I. (2013). Structures and Transformations for Model Reduction of Linear Quantum Stochastic Systems. arXiv:1308.6062.

[Petersen, 2011] Petersen, I.R. (2011). Cascade cavity realization for a class of complex transfer functions arising in coherent quantum feedback control. Automatica 47, 1757-1763.

[Petersen, 2013] Petersen, I.R. (2013). Singular perturbation approximations for a class of linear quantum systems. IEEE Trans. Automat. Contr. 58, 193-98.

[Stockton, van Handel \& Mabuchi, 2004] Stockton, J., van Handel, R., \& Mabuchi, H. (2004). Deterministic dicke-state preparation with continuous measurement and control. Phys. Rev. A 70, 022106.

[Tezak, et al., 2012] Tezak, N., Niederberger, A., Pavlichin, D., Sarma, G., \& Mabuchi, H., (2012). Specification of photonic circuits using quantum hardware description language. Phil. Trans. Roy. Soc. A 370, 5270-5290.

[Tian, 2012] Tian, L., (2012). Adiabatic state conversion and pulse transmission in optomechanical systems, Phys. Rev. Lett. 108, 153604.

[van der Schaft, 1996] van der Schaft, A. (1996). L L $_{2}$ Gain And Passivity Techniques in Nonlinear Control. Lecture Notes in Control and Information Sciences, Vol. 218, Springer.

[Ticozzi \& Viola, 2008] Ticozzi, F. \& Viola, L., (2008). Quantum Markovian subsystems: Invariance, attractivity and control. IEEE Trans. Automat. Contr., 53, 2048-2063.

[Ticozzi \& Viola, 2009] Ticozzi, F. \& Viola, L., (2009). Analysis and synthesis of attractive quantum Markovian dynamics, Automatica 45, 2002-2009.

[Wall, 1948] Wall, H.S. (1948). Analytic Theory of Continued fractions. Van Nostrand, New York.

[Wall \& Milburn, 2008] Walls, D.F. \& Milburn, G.J. (2008). Quantum Optics. Springer, Berlin, 2008.

[Willems, 1972] Willems, J.C. (1972). Dissipative dynamical systems part II: Linear systems with quadratic supply rates. Archive Rat. Mech. Anal. 45, 352-393.

[Wiseman \& Milburn, 2010] Wiseman, H. M., \& Milburn, G. J. (2010). Quantum measurement and control. Cambridge University Press, Cambridge.

[Woods, et al., 2014] Woods, M.P., Groux, R. Chin, A.W., Huelga, S.F. \& Plenio, M.B. (2014). Mappings of open quantum systems onto chain representations and Markovian embeddings. J. Math. Phys. 55, 032101.

[Yamamoto, 2013] Yamamoto, N. (2013). Decoherence-free linear quantum systems. arXiv:1210.2632v2 [quant-ph]. To appear in IEEE Transactions on Automatic Control, DOI: 10.1109/TAC.2014.2313218. 
[Zhang, et al., 2013] Zhang, J., Liu, Y.X., Wu, R-B., Jacobs, K. \& Nori, F. (2013). Non-Markovian quantum input-output networks. Phys. Rev. A, 87, 032117.

[Zhang, Chen, Bhattacharya, \& Meystre, 2010] Zhang, K., Chen, W., Bhattacharya, M., \& Meystre P. (2010). Hamiltonian chaos in a coupled BEC-optomechanical cavity system. Phys. Rev. A 81, 013802.

[Zhang \& James, 2011] Zhang, G. \& James, M.R. (2011). Direct and indirect couplings in coherent feedback control of linear quantum systems. IEEE Trans. Automat. Contr. 56, 1535-1550.

[Zhang \& James, 2012] Zhang, G. \& James, M.R. (2012). Quantum feedback networks and control: a brief survey. Chinese Science Bulletin 57, 2200-2214.

[Zhang \& James, 2013] Zhang, G. \& James, M.R. (2013). On the response of quantum linear systems to single photon input fields. IEEE Trans. Automat. Contr. 58, 1221-1235.

[Zhou, Doyle \& Glover, 1996] Zhou, K., Doyle, J., \& Glover, K. (1996). Robust and Optimal Control. Prentice-Hall, Upper Saddle River, NJ. 